\title{
Fuzzy Multicriteria Decision-Making Approach for Measuring the Possibility of Cloud Adoption for Software Testing
}

\author{
Sikandar Ali $\mathbb{D},{ }^{1,2}$ Niamat Ullah $\mathbb{D},{ }^{3}$ Muhammad Faisal Abrar $\mathbb{D},{ }^{4}$ Zhongguo Yang $\left(\mathbb{D},{ }^{5}\right.$ \\ and Jiwei Huang $\mathbb{B}^{1,2}$ \\ ${ }^{1}$ Department of Computer Science and Technology, China University of Petroleum, Beijing 102249, China \\ ${ }^{2}$ Beijing Key Laboratory of Petroleum Data Mining, China University of Petroleum, Beijing, Beijing 102249, China \\ ${ }^{3}$ Department of Computer Science University of Buner, Buner 17290, Pakistan \\ ${ }^{4}$ Department of Computer Software Engineering, University of Engineering and Technology, Mardan 23200, Pakistan \\ ${ }^{5}$ School of Information Science and Technology, North China University of Technology, Beijing, China \\ Correspondence should be addressed to Jiwei Huang; huangjw@cup.edu.cn
}

Received 20 October 2019; Revised 27 December 2019; Accepted 9 January 2020; Published 30 April 2020

Guest Editor: Rahman Ali

Copyright ( $\odot 2020$ Sikandar Ali et al. This is an open access article distributed under the Creative Commons Attribution License, which permits unrestricted use, distribution, and reproduction in any medium, provided the original work is properly cited.

\begin{abstract}
To reduce costs and improve organizational efficiency, the adoption of innovative services such as Cloud services is the current trend in today's highly competitive global business venture. The aim of the study is to guide the software development organization (SDO) for Cloud-based testing (CBT) adoption. To achieve the aim, this study first explores the determinants and predictors of Cloud adoption for software testing. Grounded on the collected data, this study designs a technology acceptance model using fuzzy multicriteria decision-making (FMCDM) approach. For the stated model development, this study identifies a list of predictors (main criteria) and factors (subcriteria) using systematic literature review (SLR). In the results of SLR, this study identifies seventy subcriteria also known as influential factors (IFs) from a sample of 136 papers. To provide a concise understanding of the facts, this study classifies the identified factors into ten predictors. To verify the SLR results and to rank the factors and predictors, an empirical survey was conducted with ninety-five experts from twenty different countries. The application value in the industrial field and academic achievement of the present study is the development of a general framework incorporating fuzzy set theory for improving MCDM models. The model can be applied to predict organizational Cloud adoption possibility taking various IFs and predictors as assessment criteria. The developed model can be divided into two main parts, ranking and rating. To measure the success or failure contribution of the individual IFs towards successful CBT adoption, the ranking part of the model will be used, while for a complete organizational assessment in order to identify the weak area for possible improvements, the assessment part of the model will be used. Collectively, it can be used as a decision support system to gauge SDO readiness towards successful CBT.
\end{abstract}

\section{Introduction}

Over the past decades, software applications are becoming complex, dynamic, component based, and distributed, therefore making software testing a challenging task [1]. Industry reports show that software of 20,000 lines of code might take seven weeks of uninterrupted execution for a thorough regression test [2]. Many software development organizations (SDOs) consider test process automation to shorten development cycle and decrease testing costs [3]. Moreover, it is time-consuming and laborious to conduct some types of testing manually like regression testing, while through test automation, they can be performed efficiently [4]. Once tests have been automated, they can be run repeatedly and quickly. Test automation also proves to be an economical method for the regression testing of software with lengthy maintenance spell [4]. However, this type of testing demands huge investment from an SDO on various testing tools and related resources which may go out of use over a period of time because technology and application advance over time. Since SDO is supposed to retain pace with the industrial changes, SDO finds it hard and unfeasible to upgrade them every time [2].

Due to the essential value-added feature of Cloud Computing (CC), paradigms such as on large resource pool, 
self-service on demand, measured service, broad network access, and rapid elasticity application development, deployment, and testing in the CC environments have been greater than before. This results in the reduction of delivery time, increased productivity, and capital and operational cost saving. Besides numerous benefits, it also postures several challenges for the quality of service (QoS) assurance including high-availability, multitenancy issues, elasticity, privacy, and security [5]. CC is regarded by many authors as a paradigm shift in computing, in lieu of an important change in the way computing services are offered, developed, attained, and paid for [6]. In practice, in this model, a Cloud service provider (CSP), serving the public, uses a pool of computing services via multitenancy. Potential clients can acquire and use these services over the Internet with automated user-friendly interfaces. Using the pay-as-you-go model, the service consumption is automatically metered [7].

At present, Cloud services are offered in at least three different models, i.e., Infrastructure-as-a-Service (IaaS), Platform-as-a-Service (PaaS), and Software-as-a-Service (SaaS). These services are typically organized in one of the four infrastructures which are public, private, hybrid, or community Cloud settings. In the near past, numerous business organizations have shown a growing interest in the adoption of Cloud services to support various corporate functions [8]. CC is one of the five most persuasive technologies across the globe and is ranked the third widely invested significant IT service in 2013 [9]. According to the latest 2018 McKinsey report, over the next three years, organizations will make a fundamental shift from developing IT to utilizing IT [10]. Companies of all sizes are moving to off-premise Cloud services, among a significant move are noticed in large enterprises. The market share of CC is increasing fast, $30 \%$ small and medium businesses (SMBs) have already acquired five or more Cloud services while $60 \%$ have acquired a minimum of one Cloud service [11]. While cost reduction is often perceived to be the main factor in adopting CC services, the report shows that quality is a key predictor for Cloud adoption, while compliance and security remain key inhibitors for acceptance, specifically for large organizations [10].

As stated above there are three basic service models of CC, i.e., IaaS, PaaS, and SaaS. However, when we want to conduct Cloud testing, there emerges a new service model called Software Testing-as-a-Service (STaaS) or simply TaaS. A new term, known as TaaS or STaaS, has become a hot topic of discussion in different research groups as well as IT businesses and the Cloud industry [12]. The term of "TaaS" was originally coined and properly introduced by Tieto (http://www.tieto.com/) in 2009 in Denmark. Afterward, TaaS has been widely accepted by both industry and academic communities owing to its advantages in utility style service models, scalable testing environment, demanded testing services, and cost reduction [12]. This model has been assimilated into commercial products like Sogeti and Tieto. TaaS in a CC environment is deliberated as a new service and business model where a TaaS service provider creates a software testing environment and provides them as-a-testing service to accomplish testing tasks and activities for SDO to test application software under-test (SUT) or a web-based system running over a Cloud infrastructure [12]. In connection to software testing, the terms "testing in the Cloud," "Cloud-based testing (CBT)," "Cloud testing," "STaaS," and "TaaS" are now widely used as synonyms.

To cope with the challenges of software testing; employing CC technology would be the best choice. While many organizations are adopting $\mathrm{CC}$ with careful assurance, testing seems to be one of the areas where they are willing to be more optimistic to move. Adoption of CC for software testing process is considered to be a safe decision because testing data does not contain any sensitive business information [2]. However, the adoption of CC for software testing is still in the initial stage [13]. The adoption rate of CC depends on the identification of novel factors. Before taking any important adoption decision, the significant factors for Cloud adoption should be ranked. Studies focusing on the adoption of CC for software testing are still lacking. Furthermore, conducting testing in the CC environment is neither cost-effective nor it is the best possible solution to all testing problems. Furthermore, not every type of software is the best candidate for testing in the Cloud [14].

SDO concerned about CBT must be conscious about the characteristics and types of software that are suitable to be tested in the Cloud environment, which enables them to better employ CBT [15]. Unit testing (particularly large regression suites), performance testing, and high-volume automated testing are the ideal choices to be migrated to the Cloud environment [14]. Unlike traditional web-based software testing, TaaS on Cloud infrastructure has several distinct requirements and unique features [12]. Some of the requirements from different perspectives are (1) the applications or SUT must be accessible online. The SUT might be a SaaS application or non-SaaS application. Additionally, this takes into account different levels of testing, for example regression or performance testing; (2) diverse deployment models of the Cloud such as private, public, hybrid, or community are used to host testing platforms and infrastructure; (3) testing of the Cloud itself [16].

Moreover, it is not straightforward to move testing to the Cloud; several quantitative and qualitative factors influence the decision to adopt CC. For example, all the traditional artifacts must be relocated to a new platform while still adhering to the original quality attributes and functionality. Questions like why tests are migrated to the Cloud, which techniques will be suitable, which test needs to be migrated, and when to migrate to the Cloud demand to be answered before proceeding with the CBT [17]. When transferring testing to the Cloud platform, the tools that are used in the course of the testing need to be switched to an innovative Cloud environment while still coinciding with the development environment. These artifacts consist of the test environment (such as testbeds and tools), test plans, testing method, test cases, test results and its documentation, and so on. Equal rewards should be reaped provided that high risks are posed while moving application testing to the CC platform [14].

1.1. Aims and Objectives. Specifically, this study explores the determinants and predictors of Cloud adoption in the 
context of software testing and develops a Cloud testing adoption assessment model (CTAAM) for decision-making towards CC adoption for software testing, specifically known as CBT. CTAAM was developed based on the input from experts' judgment using fuzzy multicriteria decision-making (FMCDM) approach. For the proposed model development, we performed a systematic literature review (SLR) for data collection by applying our customized search strings [18]. From results of the SLR study, we identify seventy influential factors (IFs) from a sample of 136 papers. To validate the SLR findings and to rank the IFs, an empirical survey was conducted in the software testing industry with ninety-five experts from 20 different countries. Based on the collected data, we performed a two-phase analysis based on FMCDM methods. We develop CTAAM model based on the twophase analysis results. For evaluation of the CTAAM two case studies were conducted in SDO. To rank the IFs, we used our developed FMCDM based framework. For this purpose, we have developed an evaluation scale with 133 items based on previous researches.

1.2. Contribution of the Study. This study contributes positively to the academic literature on the Cloud adoption in the context of software testing by identifying various criteria and is the foundation of Cloud adoption for future research in this field. Unlike other researchers, we formulate Cloud adoption as MCDM problem. Since several qualitative and quantitative factors influence decision towards Cloud adoption indicating that adoption is a kind of MCDM problem. Furthermore, unlike other researchers, we have combined the fuzzy set theory with MCDM to better handle vagueness, uncertainty, expert heterogeneity, human biasness, and subjectivity. In detail, in our framework model, the linguistic terms with parallel values in triangular fuzzy numbers (TFNs) format are used to translate the linguistic term. To handle expert heterogeneity, this study makes CTAAM capable of accumulating the decision of several experts by means of various predictors and factors as measurement criteria.

The model will do an organization assessment based on various IFs and predictors as evaluation criteria and can be applied to predict organizational Cloud adoption possibility taking various IFs and predictors as assessment criteria. The developed model can be divided into two main parts ranking and rating. To measure the success or failure contribution of the individual IFs towards successful CC adoption the ranking part of the model will be used while for a complete organizational assessment in order to identify the weak area for possible improvements the assessment part of the model will be used. Collectively, it can be used as a decision support system (DSS) to gauge SDO readiness towards successful CC adoption for CBT. The industrial contribution of the model is that it can be used as an assessment tool for SDO vendors and will indicate their weakness using an extended fuzzy version of the Motorola instrument specifically extended for this study. The models developed through FMCDM approach can handle uncertainty and vagueness in the expert judgments but they cannot identify the weak and strong areas of an organization. Therefore, our framework model in this study uses Motorola assessment tool $[19,20]$ in the case study. Similar to our previous studies [21-23], this study also prolonged the Motorola evaluation tool to a fuzzy environment by suggesting the TFNs scale for its three dimensions. The existing Motorola guidelines $[19,20]$ were appropriate for a 5-point Likert scale only. We update the guidelines to 7-point Likert and introduce TFNs instead of even numbers $(0,2,4,6$, and 10$)$. We have designed seven point linguistic scales for assessing the importance weights of 133 items. Scale development is another contribution.

Theoretical contribution of the fuzzy study is the design and structure of a general framework to improve multicriteria decision models. Other researchers and practitioners can use the proposed model structure and procedure as a template to develop FMCDM models and framework for decision-making, organizational capability improvement, and assessment for enterprises, organization, or companies in the other relevant fields. Moreover, the ranking part of the CTAAM can be adopted to design a standalone prioritization or weighting tool and to rank the impact of different risk or success factors while the assessment part of the CTAAM can be adopted to develop mini assessment tool. Collectively, it can be adopted as a DSS.

1.3. Paper Outline. The rest of the paper is organized as follows. In the second section, a brief review of the studies conducted in the domain of CBT followed by quick motivation for using fuzzy set theory is presented. Section 3 describes the research methodologies for data collection and analysis. This is then followed by results obtained through ranking and rating in Section 4. Section 5 discusses the results while Section 6 discusses the study limitations. Finally, Section 7 concludes the paper.

\section{Background and Motivation}

Cloud testing is the process of software testing based on CC technology [5]. Cloud testing can be testing the Cloud itself or TaaS over Cloud. In this study, we focus on testing applications in the Cloud, i.e., TaaS. A group of expert architects and performance testers from the UK leading web load and performance testing company (http://www. CloudTesting.com/) initiated Cloud-based testing service for load and performance testing of website and Web applications [24].

The present literature around CBT is in the form of reports and industrial white papers. However, as the academic research on CBT gains popularity, scientific research has also been growing [22, 25-29]. Researchers examine specific types of testing [28], its architecture [30], applicability through case studies [31], experiments [31], and industrial survey [25]. Other related research, focusing on overviewing [32], surveying [25,33], and summarizing the existing work [34].

The survey paper [35] poses some questions to the CC research community, which involve suitable solutions to software testing. Fernandes and Gemmer [36] have defined 
the CC paradigm and its implications for IT organizations. Bertolino et al. [37] identify various references and buzzwords. Bai et al. [24] suggest new developments in Cloud testing tools like SaaS testing, cross-Cloud testing, real-time results processing and dynamic adaptive testing, dependability testing, service-level-agreement conformance testing, and security and reliability testing. Nachiyappan and Justus [38] give an overview of Cloud testing tools. In a comparative study, they discuss various challenges to CC testing tools. Titinen [39] in their master thesis identify critical factors for software testing tools selection. They also classify testing tools into test management, execution, comparison, framework, and measurement tools. Murthy and Suma [40] conduct a study on CC testing tools, and Devasena et al. [41] propose a load testing tool for Cloud.

However, this research area is still immature [26]. For the time being, a vast majority of the preceding researches addresses the testing of the core architecture of the Cloud. Furthermore, the majority of the preceding work focuses on Cloud service evaluation. Conversely, customization of those services regarding software testing has been largely ignored in the preceding literature. What's more, few studies focus on Cloud adoption [42-48] but they are not conducted form software testing, the only exception are [22, 25]. A recent survey on testing in the Cloud [49] identified lack of a Cloud testing adoption model that guides decision-maker, when and how to move testing to the Cloud, based on various decision factors as a research gap. Therefore, our study will be an attempt to bridge some of the most important research gaps by developing a research model that is adaptable and scalable for SDO.

2.1. Motivation and Novelty. The decisions on whether to adopt Cloud as a testing tool are essential since incorrect decisions might consequence in a loss in terms of resources and efforts. A shortage of knowledge and information about a certain technology makes it uncertain and vague. Thus, unavoidably, most of the critical decisions are made under nondeterministic conditions while any carelessness may result in the dire consequences. Models and frameworks have been recommended to support the decision-makers for CC adoption, incorporating various aspects such as demand behaviors, business objectives, and quality of services, migration cost, and technical challenges. For instance, an MCDM model for ranking and selecting Cloud services includes six factors, namely, accountability, costs, assurance, agility, performance, and security [50]. Although there are numerous models for decision-making [42-48, 51-55] but they are not conducted form software testing, the only exceptions are $[14,22,25]$. Furthermore, most of them [42-48, 51-53] are not based on the fuzzy set theory. Furthermore, they are proposed for either service selection [46] or Cloud provider selection [46]. Interestingly, none of them is proposed for software testing or TaaS. The only exception is found in [14], in which authors propose a decision framework called SMART-T. SMART-T originated from Service-Oriented Architecture (SOA) Migration, Adoption, and Reuse Technique (SMART) [56] where $T$ shows testing. SMART-T contains three parts: business drivers, technical factors, and operational results. SMART-T is developed based on business drivers and technical factors. Additionally, it is not developed based on the fuzzy set theory. Moreover, we did not find any study from the perspective of CC adoption, handling vagueness, or uncertainty in experts' decision-making.

Our study will contribute to the existing literature on Cloud adoption and software testing. Unlike the preceding researchers, we formulate Cloud adoption as the MCDM problem. Since several qualitative and quantitative factors influence in decision towards Cloud adoption indicating that adoption is a kind of MCDM problem. Compared with the preceding studies, our study has the following significance. Firstly, very few studies on CBT have been conducted. Secondly, no compressive study on Cloud adoption for software can be found in the relevant literature. Therefore, this paper is the initial effort to provide new valuable discernments on the adoption of CC technology for software testing, which is considered to be an emerging research perspective in automated testing. Thirdly, besides the importance of CBT, very limited evidence in the relevant literature can be found that discuss aspects of CBT adoption through systematic theoretical evaluation and models. In fact, the application of operational research models and theories, especially technology adoption theories and the MCDM approaches for Cloud adoption in the context of software testing remains unexplored. Consequently, proposing a Cloud testing adoption assessment model (CTAAM) and linking various influential factors to its core constructs of organizational adoption intention is deliberated as a valuable tool, which is the fundamental innovation of this study. Lastly, previous studies have only utilized MCDM [45-47, 51, 57] approach in ranking the influential factors and its dimension and predicting the organizations' intention to adopt Cloud technologies but they are not conducted for software testing, the only exception is [25]. Besides, we did not find any study, handling vagueness or uncertainty in experts' decision-making as well as causal relations among the identified factors simultaneously from the perspective of CC adoption. To handle expert heterogeneity, this study develops a model capable of aggregating the decision of multiple experts by using multiple predictors and factors as measurement criteria. In our proposed framework, multiple experts can participate in both the ranking survey and rating assessment case study. Additionally, unlike the preceding studies, this study provides the experts with a self-effacing linguistic scale as presented in column 1 of Tables 1 and 2 .

\subsection{Application of Fuzzy Set Theory to Multicriteria Decision-} Making (MCDM). In real-world decision problems experts typically have to decide with imprecise, vague, or incomplete data [58]. Vagueness exists in the natural language terms, such as good or best, better than, smaller than, considerable, important, significant, not implemented, partially implemented, fully implemented, achieving, achieved, outstanding, qualified, marginally qualified, etc. $[59,60]$. Vagueness or fuzziness in data may be due to poor boundaries of scale 
TABLE 1: Linguistic scale for ranking of items.

\begin{tabular}{lc}
\hline Linguistic terms & Corresponding scale \\
\hline Not contributing at all & $(0.0,0.0,0.1)$ \\
Contributing sometime & $(0.0,0.1,0.3)$ \\
Slightly contributing & $(0.1,0.3,0.5)$ \\
Not sure & $(0.3,0.5,0.7)$ \\
Moderately contributing & $(0.5,0.7,0.9)$ \\
Strongly contributing & $(0.7,0.9,1.0)$ \\
Extremely contributing & $(0.9,1.0,1.0)$
\end{tabular}

[61]. All real numbers greater than one is a familiar example of a class of objects where boundaries are not clear or poorly defined [58]. To cope with the fuzziness usually articulated in decision data rising from the qualitative independent judgment of the experts due to the uncertainty, incomplete or vague data, the scale based on crisp numbers might be inadequate to model the real-world MCDM problems [62].

Furthermore, the influential factors are not likewise significant as the influential factors may be quite different regarding their contribution to success [59]. Thus, it is more forthright and realistic for experts to rank the IFs for example "factor F extremely contributing towards CC adoption" rather than to guess "factor " $F$ " $70 \%$ contributing towards CC adoption". Converting heterogeneous information to a single form might result in a loss of important information [63].

In practice, decision-making usually requires subjective data provided by the expert [64]. It is obvious that different experts have different preference structures, knowledge levels, and complex decision capabilities based on their experiences $[65,66]$; therefore, a good decision-making model must tolerate vagueness or ambiguity in expert judgment $[60,65]$. Numerous researchers have recommended incorporating fuzzy set theory $[54,55,58-67]$ dispensing subjectivity in decision-making by human experts. Fuzzy statistics in FMCDM methods are represented by fuzzy numbers via fuzzy sets. In fuzzy sets, the degree of association is allocated to objects in their universe of discourse. An object in this approach can fit some sophisticated classes of objects with only a partial association [67].

\subsection{Fuzzy Multicriteria Decision-Making (FMCDM).} Zadeh [67] is the first researcher to incorporate "fuzzy sets theory" and proposed the MCDM approach for subjective decision-making. It has been widely adopted to model uncertainties in human decision-making. It also meritoriously resolves uncertainties in collecting data for MCDM. Several researchers have identified that the subjective vagueness of expert judgment can be dispensed by integrating the fuzzy set theory $[59,60,65,66]$ with MCDM. In a fuzzy set, linguistic terms are used to denote fuzzy variables that are then translated to the corresponding numerical values using the predefined linguistic scale $[65,66]$.

2.4. Fuzzy Numbers and Fuzzy Sets. A fuzzy set has a membership function which assigns a score of association to objects within its universe of information between 0 and 1 .
TABLE 2: Linguistic scale for rating of items.

\begin{tabular}{lc}
\hline Linguistic term & Corresponding scale \\
\hline Very poor implementation & $(0.0,0.0,0.1)$ \\
Poor implementation & $(0.0,0.1,0.3)$ \\
Weak implementation & $(0.1,0.3,0.5)$ \\
Fair implementation & $(0.3,0.5,0.7)$ \\
Marginally implemented & $(0.5,0.7,0.9)$ \\
Fully implemented & $(0.7,0.9,1.0)$ \\
Outstanding & $(0.9,1.0,1.0)$ \\
\hline
\end{tabular}

Figure 1 shows the $x$ coordinates of the three vertices $l$ lower, $m$ central, and, $u$ upper of $\mu \mathrm{A}(\alpha)$ in a fuzzy set $\mathrm{A}$. Let $U$ is a universal set having items $\{\alpha\}$ then, a fuzzy set $\widetilde{A}$ in the universe of information $U$ defined by its membership function is as follows:

$$
\mu_{A}(\alpha): U \longrightarrow[0,1]
$$

which assigns to each $\{\alpha\}$ a score of association to $\widetilde{A}$ in the interval $[0,1]$. Here $\mu_{A}(\alpha)=$ score of membership of $\alpha$ in $\widetilde{A}$, assign values in range of 0 to 1 , i.e.,

$$
\mu \bar{A}(\alpha) \in[0,1] .
$$

A fuzzy set can also be represented by a continuous membership function $\mu \bar{A}(\alpha)$.

$$
\mu \bar{A}(\alpha)=\left\{\begin{array}{cc}
0, & \text { if } \alpha \leq 1, \\
\frac{\alpha-1}{m-l}, & \text { if } l \leq \alpha \leq m, \\
\frac{u-\alpha}{u-m}, & \text { if } m \leq \alpha \leq u, \\
0, & \text { if } \alpha \geq u,
\end{array}\right\} .
$$

2.5. Linguistic Scale. The conventional scaling approaches face difficulties in labeling the criteria that are overly complicated or hard to define [67]. To counter the consequences of fuzziness the linguistic scale is used. Linguistic terms with parallel TFNs offer practical means for dispensing incidents of subjectivity. The appropriateness of substitutes versus criteria and the importance weights of criteria are assessed using a linguistic scale with corresponding fuzzy numbers [59, 60]. Several researchers $[21-23,59,60,65,66]$ recommend the linguistic scale for describing such situations.

Inspired by the above-reported studies, in this paper, the linguistic terms with parallel TFNs scores are used to reproduce the information. Precisely, we have designed seven Likert linguistic scales for ranking IFs via 133 items. Seven Likert linguistic scales as presented in Table 1 were used to rank the significance of the identified factors via its items found via SLR in phase 2.

Likewise, seven linguistic scales as presented in Table 2 grounded on Motorola evaluation tool are designed. The case study companies were asked to rate the level of 


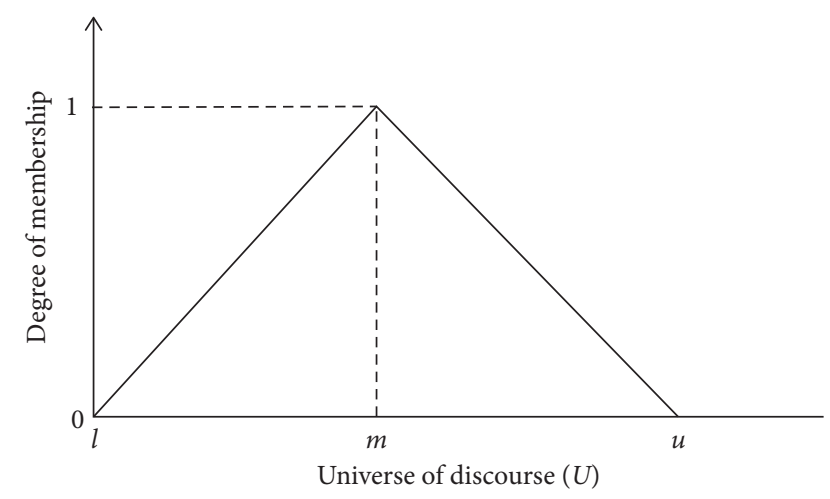

Figure 1: Coordinates of Triangular fuzzy numbers.

implementation of items across the three dimensions of Motorola evaluation tool [21], i.e., results, deployment, and approach as presented in Table 3.

\section{Research Methodology}

In terms of the proposed research model, we have developed it by including empirical research methodology based on a mixture of quantitative and qualitative research techniques for data collection and a two-phase FMCDM approach for analysis as deliberated in the following subsections.

Phase\#1: to identify critical influential factors (CIFs), for data collection at the early stage, SLR was used.

Phase\#2: to rank the identified CIFs using fuzzy FMCDM and at the same time to validate the SLRs findings, a questionnaire survey was carried out.

Phase\#3: based on the inputs from the questionnaire survey and SLR the CTAAM was developed in phase 3.

Phase\#4: to assess the CTAAM through the FMCDM approach and at the same time to evaluate its effectiveness in real-world environment case study was executed.

3.1. Systematic Literature Review (SLR). This study conducts SLR [68] to find the critical factors of the adoption of CC for software testing. Before conducting the SLR, we design a review plan known as a protocol. We have published the SLR protocol with initial results in a conference paper [18].

3.1.1. Review Plan. It reduces researchers' bias and enhances review accuracy and repeatability [68]. Particularly, it outlines rationale for the study, search strategies, study questions, literature inclusion/exclusion and quality assessment checklists, and data extraction and synthesis plans [68]. Figure 2 illustrates the main phases of the SLR process.

Construction of Search Terms. In terms of the searching phase, the scope of the study is acknowledged as follows:

Population. Primary studies on "Cloud-based testing," "testing in the Cloud," "Cloud testing," "testing-as-aservice," and "TaaS."
Intervention. Published primary studies reporting success factors for Cloud-based software testing.

Outcomes of Relevance. List of factors related to the CBT adoption.

Experimental Design. Any forms of empirical study. The below example shows the aforementioned parts: [What factors/challenges] $\longrightarrow$ "INTERVENTION" In [Cloud software testing] $\longrightarrow$ "POPULATION" Which impacts upon

[Adoption of Cloud] $\longrightarrow$ "OUTCOMES OF RELEVANCE"

\section{KEYWORDS_ABSTRACT_TITLE}

(Software OR Application OR program) AND (Test* OR Validat* OR verification OR maintenance OR accepting) AND (Cloud OR Cloud computing OR TaaS OR Testing as a service) AND (factors OR motivators OR drivers OR elements OR parameters OR characteristics OR adopt* decision OR criteria OR "lesson learned")

Search plan: We used an automatic search method for publication selection because manual search is not easy for digital libraries where the numbers of available documents are over several thousands. The final search string is given below.

Literature Selection: In this step, the investigators have to list sources to be searched, inclusion, and exclusion and quality criteria.

Selection of sources: Following the guidelines for SLR [68], we selected seven search engines/databases related to the study subject to run an exhaustive search [68]. Databases are chosen based on the characteristics, such as coverage of indexed articles (journals, conferences, or books), availability of the study, and importance to the software engineering area. Following are the seven databases, we selected.

(1) ACM_digital library http://www.acm.org/

(2) Scholar_Google https://scholar.google.com

(3) IEEE_Xplore-http://ieeexplore.ieee.org/

(4) Cite_Seer-http://citeseer.ist.psu.edu/

(5) Science_Direct-http://www.sciencedirect.com/

(6) Springer_Link-http://link.springer.com/

(7) Wiley_online library-https://onlinelibrary.wiley. $\mathrm{com} /$

Inclusion and Exclusion Criterion: An inclusion and exclusion criterion is defined to verify the importance and usefulness of each study. The relevant criteria are listed in Table 4.

Quality Criteria: The quality checks are listed in Table 5. The checklist will be scored based on three possible answers; yes, partial, and no. If any of the checks are not relevant to any articles, then it will be excluded from the evaluation of that particular study only. Those studies that will not provide the basic information about their research methods will score less than 50\% in quality assessment and will be excluded. 
TABLE 3: Key evaluation dimensions of Motorola assessment tool.

\begin{tabular}{|c|c|c|c|}
\hline Score & Approach & Deployment & Results \\
\hline Very poor & $\begin{array}{l}\text { (i) Items not evident OR } \\
\text { (ii) No ability to implement OR } \\
\text { (iii) No management indebtedness of } \\
\text { the need OR }\end{array}$ & $\begin{array}{l}\text { (i) No department of the organization } \\
\text { can use the items OR } \\
\text { (ii) No department of the organization } \\
\text { express interest }\end{array}$ & (i) No change \\
\hline Poor & $\begin{array}{l}\text { (i) Poor organizational skills OR } \\
\text { (ii) Poor management recognition of } \\
\text { the need OR } \\
\text { (iii) Poor organizational commitment }\end{array}$ & $\begin{array}{l}\text { (i) Some department of the organization } \\
\text { uses the item OR } \\
\text { (ii) Some department of the organization } \\
\text { express interest }\end{array}$ & (i) Ineffective \\
\hline Weak & $\begin{array}{l}\text { (i) Supportive action for the items } \\
\text { starts OR } \\
\text { (ii) Some departments of the } \\
\text { organization can instrument the items } \\
\text { OR } \\
\text { (iii) Management starts recognizing } \\
\text { the needs }\end{array}$ & $\begin{array}{l}\text { (i) Fragmented or inconsistent use of } \\
\text { item OR } \\
\text { (ii) Implemented in some departments } \\
\text { of the organization OR } \\
\text { (iii) Item use subject to experimenting in } \\
\text { some departments of the organization }\end{array}$ & $\begin{array}{l}\text { (i) Inconsistent results OR } \\
\text { (ii) Spotty results OR } \\
\text { (iii) Indication of effectiveness only } \\
\text { for some departments of the } \\
\text { organization }\end{array}$ \\
\hline Fair & $\begin{array}{l}\text { (i) Process for items putting into } \\
\text { practice defined } \\
\text { (ii) Numerous supportive actions for } \\
\text { the items take place OR } \\
\text { (iii) Widespread commitment but no } \\
\text { full assurance by management }\end{array}$ & $\begin{array}{l}\text { (i) Item use subject to experimenting in } \\
\text { some major departments of the } \\
\text { organization OR } \\
\text { (ii) Uniformity in use or less fragmented } \\
\text { use OR } \\
\text { (iii) Used in some major departments of } \\
\text { the organization OR }\end{array}$ & $\begin{array}{l}\text { (i) Unpredictable results for other } \\
\text { departments of the organization OR } \\
\text { (ii) Reliable and positive results for } \\
\text { some important departments OR }\end{array}$ \\
\hline $\begin{array}{l}\text { Marginally } \\
\text { Implemented }\end{array}$ & $\begin{array}{l}\text { (i) Supportive items took in place OR } \\
\text { (ii) Some managers become } \\
\text { preemptive and assure to implement } \\
\text { OR } \\
\text { (iii) Items implementation across } \\
\text { every part of the organization OR }\end{array}$ & $\begin{array}{l}\text { (i) Item use subject to experimenting in } \\
\text { several departments of the organization } \\
\text { OR } \\
\text { (ii) Item used in many departments of } \\
\text { the organization OR } \\
\text { (iii) Item mostly stable across various } \\
\text { departments of the organization }\end{array}$ & $\begin{array}{l}\text { (i) Positive quantifiable results in } \\
\text { maximum departments OR } \\
\text { (ii) Constantly positive results over } \\
\text { time throughout the organization }\end{array}$ \\
\hline Implemented & $\begin{array}{l}\text { (i) Item established as an } \\
\text { indispensable part of the process OR } \\
\text { (ii) Most of the administration is } \\
\text { proactive OR } \\
\text { (iii) Supporting items facilitates and } \\
\text { encourages the use of practice OR } \\
\text { (iv) The entire management is } \\
\text { committed OR }\end{array}$ & $\begin{array}{l}\text { (i) Item use subject to experimenting in } \\
\text { nearly every department of the } \\
\text { organization OR } \\
\text { (ii) Installed in nearly every department } \\
\text { of the organization OR } \\
\text { (iii) Constant use across nearly every } \\
\text { department of the organization }\end{array}$ & $\begin{array}{l}\text { (i) Constantly positive outcomes } \\
\text { over time across nearly every } \\
\text { department OR } \\
\text { (ii) Positive measurable outcomes in } \\
\text { nearly all departments }\end{array}$ \\
\hline Outstanding & $\begin{array}{l}\text { (i) Management provides enthusiastic } \\
\text { leadership and commitment OR } \\
\text { (ii) Organizational excellence in the } \\
\text { practice recognized even outdoor the } \\
\text { firm }\end{array}$ & $\begin{array}{l}\text { (i) Item use subject to experimenting in } \\
\text { every department of the organization } \\
\text { OR } \\
\text { (ii) Consistent use with the passage of } \\
\text { time throughout the organization OR } \\
\text { (iii) Consistent setup in every part of the } \\
\text { organization }\end{array}$ & $\begin{array}{l}\text { (i) Requirement exceeded OR } \\
\text { (ii) Guidance sought by others OR } \\
\text { (iii) Constantly world-class } \\
\text { outcomes }\end{array}$ \\
\hline
\end{tabular}

Data extraction: Primary reviewers will independently review all papers. In the case of needs for guidance, the secondary reviewers will be approached.

Execution of the review: The review was conducted from June 2017 to September 2018. To support the protocol trial, we used the EndNote X7 tool for the control group of primary studies and paper selection and storage. Moreover, Microsoft Access was used to manage the data extraction and quality assessment.

3.2. Empirical Survey. To rank the identified CIFs using FMCDM and at the same time to validate the SLR's findings, a questionnaire survey was carried out in the software development industry. Questionnaire survey serves as an appropriate method of gathering tacit quantitative and qualitative facts [69]. The process of a questionnaire survey can be divided into two phases, designing and sampling. In the design phase, a set of questions are designed to be answered by the sample. In the sampling phase, the investigator discovers, lists, selects, and invites suitable experts to contribute in the questionnaire survey [70]. Both are described briefly in the below subsections.

3.2.1. Implementing Online Questionnaire Survey. Surveys can be developed using either commercial or selfdeveloped tools. We have used Google drive where we typed 


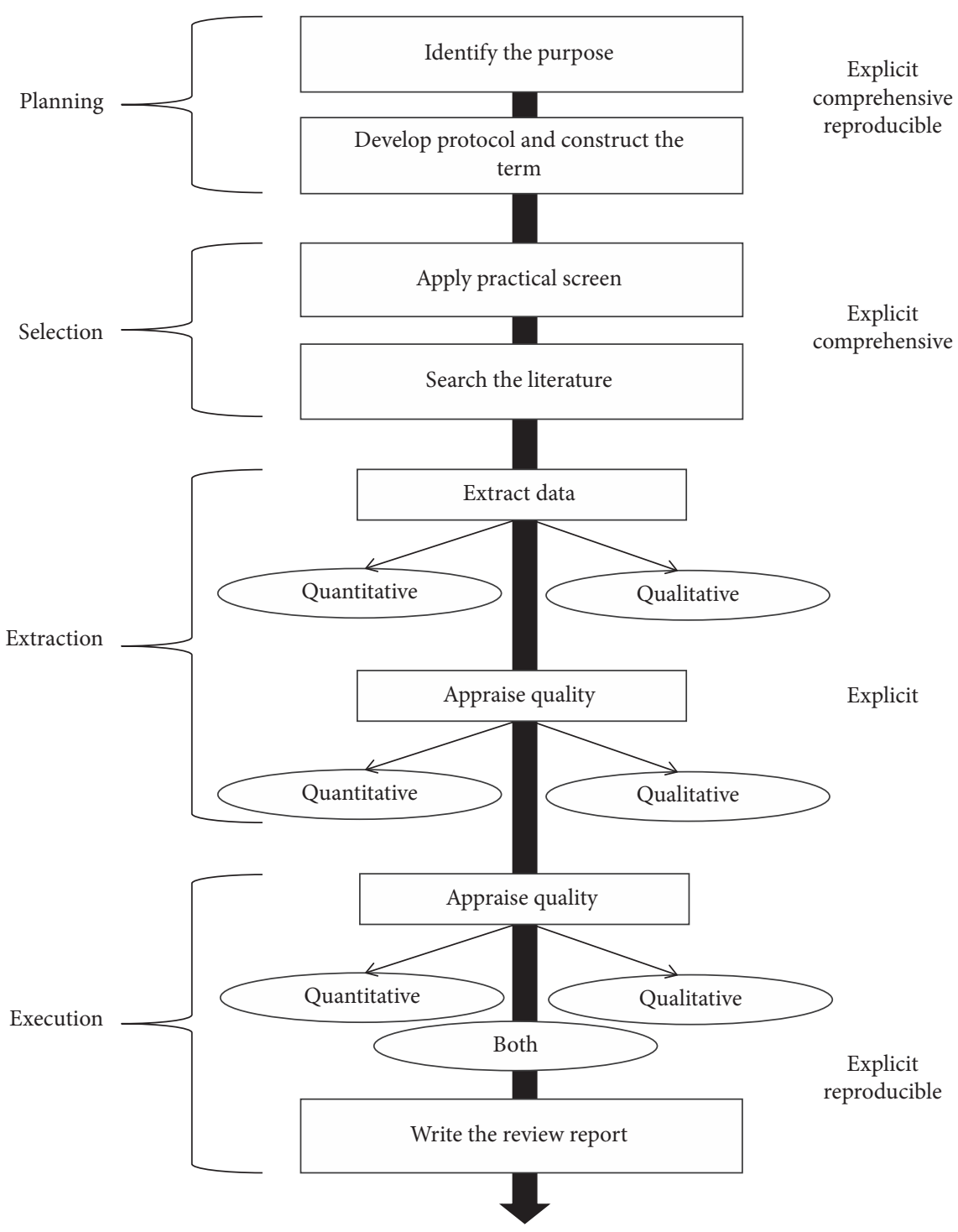

FIgURE 2: Various stages in the SLR process.

questions and required scripts and web pages came out as results. It is a commercially available online application. It also helps in collecting responses from participants, because it facilitates resending of the link through e-mail in case of no response. The questionnaire design was piloted through six members of our laboratory and necessary changes were made accordingly.

3.2.2. Data Gathering. Survey inquiry is deliberated as a suitable method of gathering tacit qualitative and quantitative data [69]. The questions of the questionnaire are of two types: open-ended, also called subjective, and close-ended, called objective. The subjective questions allow a variety of answers from the respondents, while for objective, only one choice can be chosen from the available choices. This method of data gathering assists in reducing the threat of bias relating to the investigator's prejudices. It encourages the respondent to give her/his view regarding a specific question $[69,70]$. Before a questionnaire, each participant was sent a questionnaire invitation letter. This letter outlined the main theme of the questionnaire survey, the expected duration, and measures that could be taken to ensure privacy and confidentiality. We concentrate on obtaining the linguistic weight for the influential factors (items) because not all the factors are equally important for the adoption of CC in connection to software testing.

3.3. Analysis Approach. For analysis purposes, we use the FMCDM framework presented in the next section.

3.3.1. FMCDM Framework for Measuring the Possibility of Cloud Adoption for Software Testing. This paper aims to increase empirical knowledge of CC adoption for software testing. Recently, the use of MCDM approaches has been increasing in information science and software engineering. In this paper, we successfully adopt FMCDM for the evaluation and assessment of CBT in the software industry. For 
TABLE 4: Inclusion criteria/exclusion criteria.

\begin{tabular}{|c|c|c|}
\hline \multicolumn{3}{|c|}{ Inclusion criteria } \\
\hline 1 & & Studies that describe factors for cloud-based software testing (CBT) \\
\hline 2 & & Studies that describe the evaluation of tools, methods, processes, and techniques for CBT. \\
\hline 3 & & Studies that describe advice/strength/weakness of the CBT framework \\
\hline 4 & & Studies that describe lessons learned in adopting CBT tools/services \\
\hline 5 & & Studies that describe techniques/process for migrating software testing to cloud platform \\
\hline 6 & & Studies that describe criteria for what and when to shift software testing to cloud platform \\
\hline 7 & & Studies that describe solutions to the challenges in CBT testing domain \\
\hline 8 & & Studies that evaluate CBT service provider practical capabilities for automatic software testing \\
\hline \multicolumn{3}{|c|}{ Exclusion criteria } \\
\hline 1 & & Studies written in a language other than English \\
\hline 2 & Studies tha & o not mention practices/solutions/challenges/tools/methods or techniques in automatic software testing in the Cloud \\
\hline 3 & & Studies conducted in a domain other than software engineering \\
\hline 4 & & Studies with less than four pages of contents \\
\hline 5 & & Studies published in more than one sources \\
\hline 6 & & Studies which get a low score on the quality checklist \\
\hline
\end{tabular}

TABLE 5: Quality criteria.

\begin{tabular}{lc}
\hline S. no & Quality criteria \\
\hline 1 & Is it clear how the factors that influence the adoption are identified? \\
2 & Are the findings supported by extensive data? \\
4 & Are the objectives clearly stated? \\
5 & Are the experimental or observational units adequately described? \\
6 & Is the paper based on based on some standard theory or model? \\
7 & Are negative findings presented? \\
8 & Are the implications clearly stated? \\
9 & Are all the data proposed in the data extraction form possible to extract? \\
11 & If there is a control, what are these variables that may affect study results? \\
12 & Is the study context well defined? \\
& Are the testing strategies formally described?
\end{tabular}

the stated research model development, we have incorporated a five-phase empirical research methodology using a mix of qualitative and quantitative research techniques for data collection and MCDM approach for model development and evaluation. An FMCDM framework model as shown in Figure 3 was developed to rank and rate the identified alternative criteria by assembling multiple experts in decision-making as deliberated below.

Phase\#1: Identification of the Influential Factors via SLR. To find the influential factors for the adoption of CC for CBT, the review protocol was executed to select papers through five distinct stages. In the first stage, after the selection of the databases, the search string was applied to the selected sources and we got 1,650 research articles for further process. The number of returned papers in each database is shown in Table 6 . In the second stage, we read the titles and keywords and apply inclusion criteria. In the third stage, we read the abstracts and conclusions and apply exclusion criteria. At stage-3, we also excluded 29 studies that were repeated across different databases. In the fourth phase, we read the full text of the remaining articles and applied quality criteria along with inclusion/exclusion, which results in the studies to be described in the review. After the data extraction and execution stage, we extract a list of quotes from the sample of 136 papers, where each quote described a list of influential factors. The primary reviewers in conjunction with the secondary reviewers reviewed these quotes to classify these quotes into different categories. Initially, 82 categories were identified; these were reviewed and merged into 70 different factors.

\subsubsection{Classification of the Identified Factors into Different} Dimensions. This study presents the participants with the ease to understand linguistic terms, parameterized by TFNs to precise the individual disagreement or agreement about the importance of identified predictors (main criteria) and factors (subcriteria). We are concerned about finding the ranks because all the criteria (factors and predictors) are not equally important. To rank the predictors, we divided it into small factors called subcriteria.

After data collection through SLR, the identified factors (also called subcriteria in this section) are framed into twelve major variables, ten independent (also called main criteria in this section), one mediator, and one dependent variable. Out of seventy influential factors, eleven were identified to have negative effect on the Cloud adoption, these are term as barriers. The fifty-nine factors are distributed into ten main 


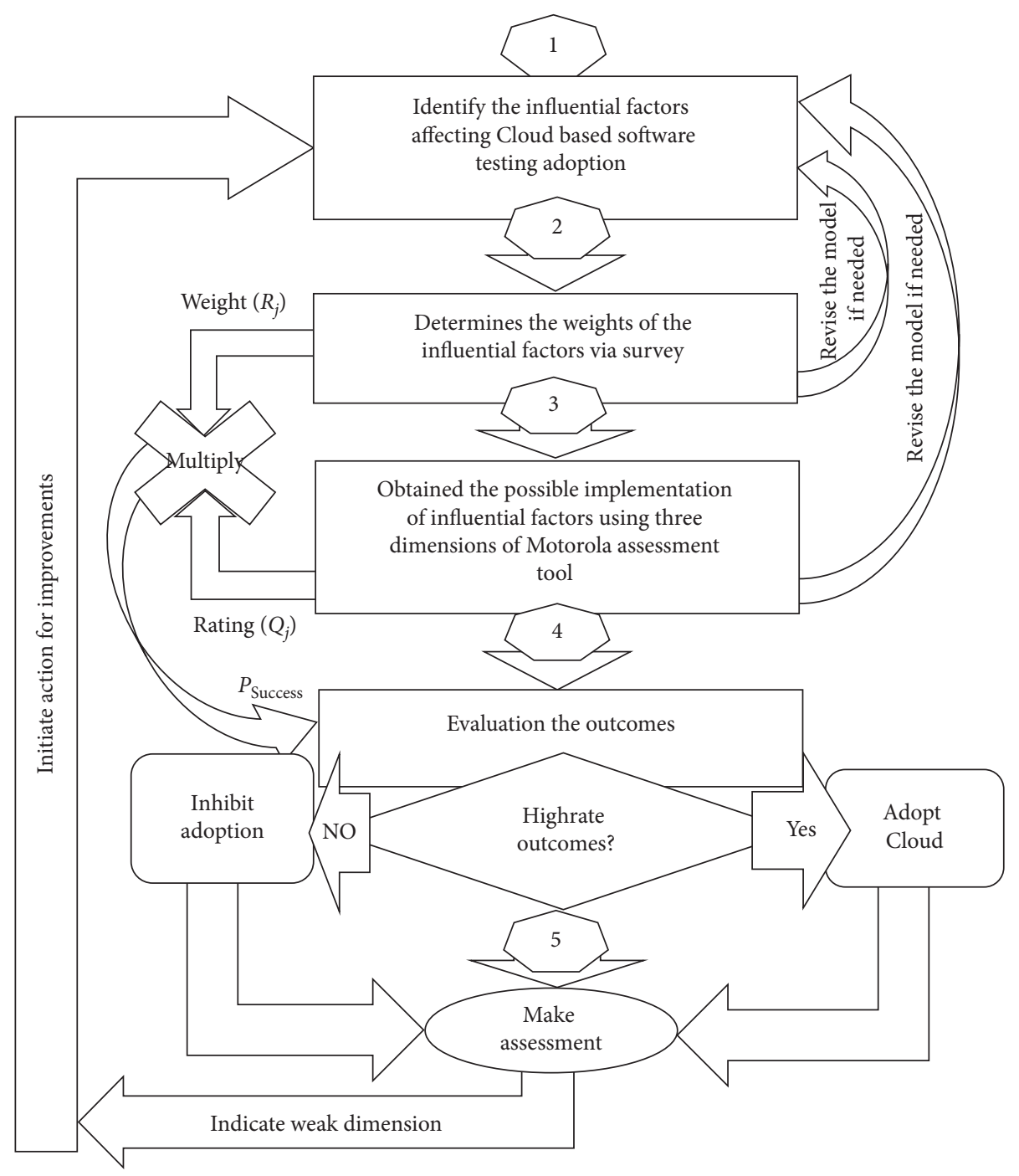

FIGURE 3: FMCDM model for measuring the possibility of successful Cloud adoption for software testing.

Table 6: Papers included/excluded during different phases.

\begin{tabular}{lcccc}
\hline Search venue & After phase 1 & After phase 2 & After phase 3 & After phase 4 \\
\hline Science direct & 177 & 83 & 80 & 14 \\
ACM & 123 & 55 & 229 & 17 \\
IEEE xplore & 842 & 250 & 21 & 76 \\
Springer link & 77 & 32 & 06 & 13 \\
CiteSeer & 63 & 07 & 21 & 03 \\
Google scholar & 400 & 58 & 03 & 12 \\
Wiley & 13 & 03 & 464 & 01 \\
Total & 1650 & 533 & & 136 \\
\hline
\end{tabular}

variables that are supposed to positively affect the adoption decision while the 11 barriers collectively form one variable that is supposed to negatively affect adoption decision (Figure 4).

3.3.3. Scale Development and Validation. The questions and the items to measure factors for this study have been borrowed from the preceding research on CC adoption. The scales used to collect the survey data about various predictors and factors used for this study are illustrated in Appendix-A in Supplementary materials. The finest means for assuring content validity is to select items from the earlier confirmed scale [71]. Therefore, in this study, we try to borrow items from preceding research to show generalized insights. To further improve the questionnaire design and contents, the questionnaire was sent to two experts in this field and two experts in academia conducting research in the relevant field. Their comments were incorporated accordingly. 


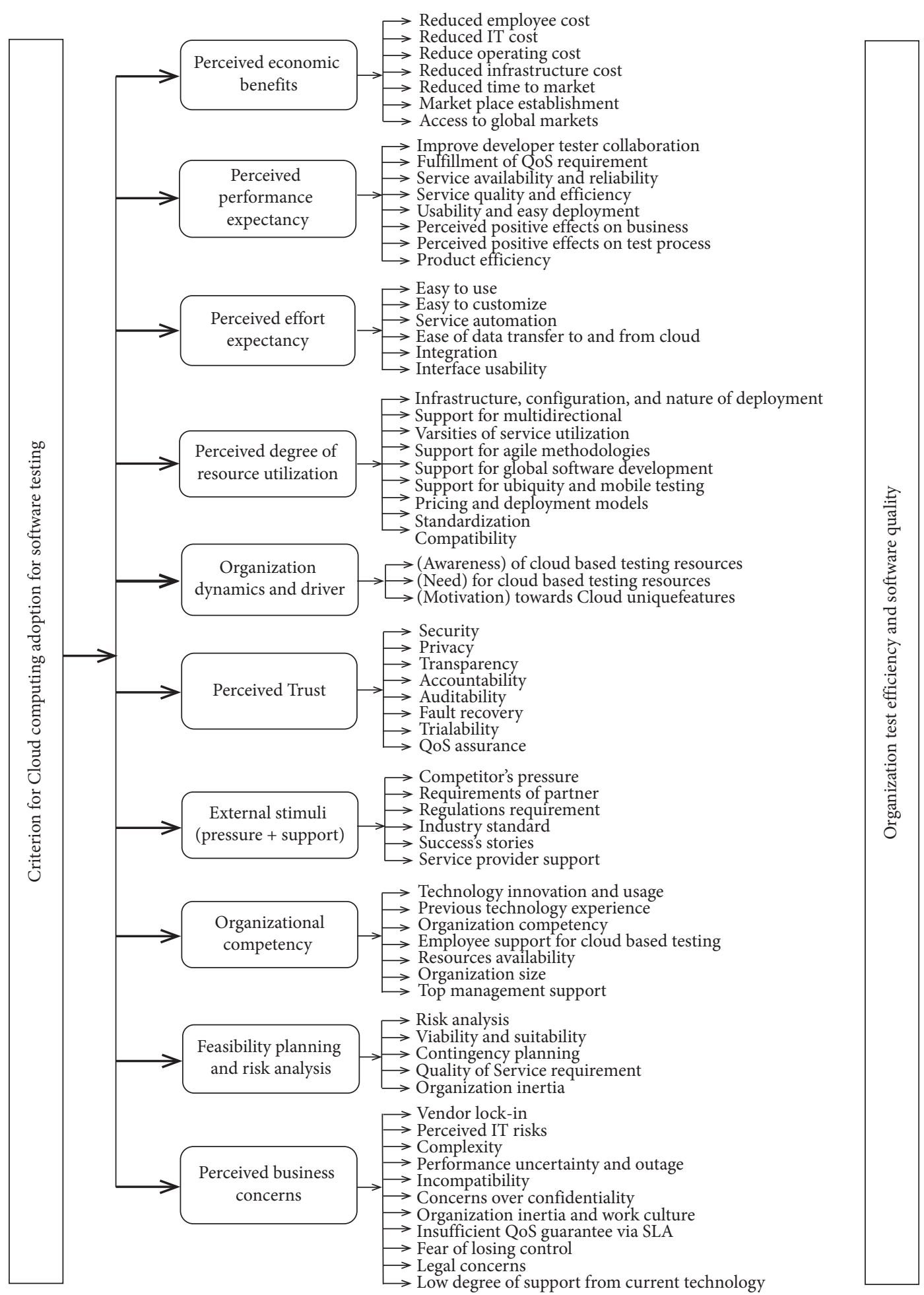

Figure 4: A conceptual model of Cloud adoption: predictors and factors.

Based on the outcomes of SLR, we have identified ten main criteria and seventy subcriteria as illustrated in Figure 4. These criteria were measured through one hundred and thirty-three items in an expert-based questionnaire survey. The items are presented in Appendix-A in Supplementary materials.
Phase\#2: Weighting the Influential Factors via Empirical Survey. To rank the influential factors and validate the findings of SLR, an empirical questionnaire survey was performed. Survey investigation is considered an appropriate method for collecting tacit quantitative and qualitative data [69]. SLR results were used as input to the 
questionnaire. The importance weights of main and subcriteria are assessed through a linguistic scale denoted by TFNs $[59,60]$. In this study, we incorporate the fuzzy scores both in the weighting survey (see Table 1) and implementation case study (Table 2). Specifically, based on TFNs as shown in the second column of Tables 1 and 2, we translated the expert opinions for processing through the FMCDM framework.

3.3.4. Obtaining the Significant Weight of the Influential Factors via FMCDM. The process for computing the significant weights of items is described in the following steps:

Step 1: Generate a decision matrix $\widetilde{A}$ for the significance weights of items $\left(I_{b}, b=1,2,3, \ldots, n\right)$. The participants of the survey $\left(E^{a}, a=1,2,3, \ldots, m\right)$ are then asked to give their subjective judgment about the significant weight of each item $\left(\widetilde{\alpha}_{b}^{a}\right.$ where $I$ represents item, $b$ represents item's number, and $a$ represents an expert's number) through the linguistic variables as given in Table 1, e.g.,

$$
\begin{aligned}
& E^{1} E^{2} E^{3} \cdots E^{m} \\
& I_{1}\left[\begin{array}{ccccc}
\tilde{\alpha}_{1}^{1} & \tilde{\alpha}_{1}^{2} & \tilde{\alpha}_{1}^{3} & \cdots & \tilde{\alpha}_{1}^{m}
\end{array}\right. \\
& I_{2} \quad \tilde{\alpha}_{2}^{1} \tilde{\alpha}_{2}^{2} \tilde{\alpha}_{2}^{3} \ldots \tilde{\alpha}_{2}^{m} \\
& \tilde{A}=I_{3} \quad \tilde{\alpha}_{3}^{1} \tilde{\alpha}_{3}^{2} \tilde{\alpha}_{3}^{3} \ldots \tilde{\alpha}_{3}^{m}, \quad a=1,2, \ldots, m ; b=1,2, \ldots, n \text {. } \\
& \vdots \quad \vdots \quad \vdots \quad \vdots \quad \ddots \quad \vdots \\
& I_{n}\left[\begin{array}{lllll}
\tilde{\alpha}_{n}^{1} & \widetilde{\alpha}_{n}^{2} & \widetilde{\alpha}_{n}^{3} & \cdots & \widetilde{\alpha}_{n}^{m}
\end{array}\right]
\end{aligned}
$$

where $m$ represents the total number of experts and $n$ represents the total number of items and, $\widetilde{\alpha}_{b}^{a}=\left(l \widetilde{\alpha}_{b}^{a}, m \widetilde{\alpha}_{b}^{a}, u \widetilde{\alpha}_{b}^{a}\right)$ represents the fuzzy weight of the $b$ th items given by ath respondents.

Step 2: As the independent assessment of each expert, vary according to his or her, perception, knowledge, role, and experience of the subject matter. Thus, we calculate the average score to cumulative the fuzzy importance of each item by $m$ experts.

$$
\widetilde{\omega}_{b}=\frac{1}{m}\left[\sum_{a=1}^{m} \widetilde{\alpha}_{b}^{a}\right],
$$

where $\widetilde{\omega}_{b}=\left(l \omega_{b}, m \omega_{b}, u \omega_{b}\right)$ represents the cumulative fuzzy importance weight of the $b$ th item.

Step 3. The cumulative fuzzy importance $\widetilde{\omega}_{b}$ is used to calculate the best nonfuzzy performance (BNF) value $\mathrm{BNP}_{W_{b}} \cdot \mathrm{BNP}_{W_{b}}$ can be obtained using

$$
\mathrm{BNP}_{W_{b}}=\frac{\left[\left(u w_{b}-l w_{b}\right)+\left(m w_{b}-l w_{b}\right)\right]}{3}+l w_{b},
$$

where $\mathrm{BNP}_{W_{b}}$ denotes the best nonfuzzy performance (BNP) score for the TFN $\widetilde{\omega}_{b}$ while $W_{b}$ is the significant weight of the $b$ th item in the crisp numeral format. The local ranks will be obtained through $W_{b}$.

Step 4. After the defuzzification of TFNs in step 4, crisp numbers are calculated and normalized through (7) while the items global ranks $R_{b}$ will be obtained through equation (7):

$$
R_{b}=\frac{W_{b}}{\sum_{b=1}^{n} W_{b}},
$$

where $R_{b}$ denotes the normalized importance of the $b$ th item such that $\sum_{b=1}^{n} R_{b}=1$.

Step 5. Since each item measures different aspects of the subcriteria, we incorporate the average score method to cumulative the best fuzzy importance $W_{f}$ of each subcriteria (factors).

$$
W_{f}=\frac{\sum_{b=1}^{k} W_{b}}{k}
$$

where $W_{b}$ represents the BNP weight of each item in the particular factor (subcriteria), $k$ represents the total number of items measuring that factors while $\sum_{b=1}^{k} W_{b}$ is the aggregate $\mathrm{BNP}$ weight of all the items in that factor.

\subsubsection{Computing the Significant Weight of the Predictor via FMCDM}

Step 6. Using $W_{b}$ (BNP weight of items), we can obtain $W_{p}$, BNP weight of predictor using

$$
W_{p}=\sum_{b=1}^{h} W_{b},
$$

where $W_{b}$ represents the BNP weight of each item in the particular predictor, while $h$ represents the total number of items in that predictor. We also obtain and normalize the crisp number for each predictor and each factor within predictor using equations (10) and (11):

$$
R_{f}=\frac{W_{f}}{\sum_{f=1}^{h} W_{f}},
$$

where $R_{f}$ represents the normalized significance weight of the $b$ th factor such that $\sum_{f=1}^{h} R_{f}=1$.

$$
R_{p}=\frac{W_{f}}{\sum_{b=1}^{n} W_{b}},
$$

where $\sum_{b=1}^{n} W_{b}=W_{t}$ sum of the weight of all items and $R_{p}$ represents the normalized significance weight of the $b$ th predictor such that $\sum_{b=1}^{n} R_{b}=1$.

Phase\#3: Rating of the Influential Factors via Case Study. To assess the CTAAM through MCDM approach and at the same time evaluate its effectiveness in real-world 
environment, two case studies were executed. Case study can provide adequate evaluation in real-world industrial environments [72]. Similar to the questionnaire survey, here we also translate the expert's judgment to TFNs format but unlike them, we use the extended version of the Motorola evaluation tool [21] to evaluate the SDO readiness towards Cloud adoption for CBT. For the assessment of influential factors and to find the implementation of various dimensions in the assessment case study, we also incorporate the fuzzy set theory. Seven linguistic scales as illustrated in Table 2 based on the dimension of Motorola evaluation tool design for case companies to rate factors across the three dimensions of Motorola evaluation tool [73] such as approach, deployment, and results as presented in Table 3.

3.3.6. Obtaining the Readiness of the Software Testing Organizations for Cloud Adoption via FMCDM. The procedures for calculating the degree of implementation of the items in particular organizations are described in the following steps:

(i) Step 1: Generate three matrices $\widetilde{B}_{A}, \widetilde{B}_{D}$, and $\widetilde{B}_{R}$ for the rate of implementation of the items $\left(I_{b}, b=1,2,3, \ldots\right.$, $n)$. A, $D$, and $R$ denotes the three dimensions of the Motorola evaluation tool as presented in Table 3 . The respondents of the survey $\left(E^{a}, a=1,2,3, \ldots, m\right)$ are asked to give their expert judgment about the degree of implementation of the items in their respective organizations following the guidelines of Motorola evaluation tool as illustrated in Table 3, by using the linguistic scale as presented in Table 2, e.g.,

$$
\begin{aligned}
& \begin{array}{lllll}
E^{1} & E^{2} & E^{3} & \cdots & E^{m}
\end{array} \\
& I_{1}\left[\begin{array}{lllll}
\tilde{\beta}_{1}^{1} & \tilde{\beta}_{1}^{2} & \tilde{\beta}_{1}^{3} & \cdots & \tilde{\beta}_{1}^{m}
\end{array}\right] \\
& \begin{array}{l|lllll}
I_{2} & \tilde{\beta}_{2}^{1} & \tilde{\beta}_{2}^{2} & \tilde{\beta}_{2}^{3} & \cdots & \tilde{\beta}_{2}^{m}
\end{array} \\
& \widetilde{B}_{A}=I_{3}\left|\begin{array}{ccccc}
\tilde{\beta}_{3}^{1} & \tilde{\beta}_{3}^{2} & \tilde{\beta}_{3}^{3} & \ldots & \widetilde{\beta}_{3}^{m}
\end{array}\right|, \quad a=1,2, \ldots, m ; b=1,2, \ldots, n \text {, } \\
& \vdots \quad \begin{array}{ccccc}
\vdots & \vdots & \vdots & \ddots & \vdots
\end{array} \\
& I_{n}\left[\begin{array}{lllll}
\tilde{\beta}_{n}^{1} & \widetilde{\beta}_{n}^{2} & \tilde{\beta}_{n}^{3} & \cdots & \widetilde{\beta}_{n}^{m}
\end{array}\right] \\
& \begin{array}{lllll}
E^{1} & E^{2} & E^{3} & \cdots & E^{m}
\end{array} \\
& \widetilde{B}_{D}=I_{3}\left[\begin{array}{c}
I_{1} \\
I_{2} \\
\vdots \\
I_{n}
\end{array}\left[\begin{array}{ccccc}
\tilde{\beta}_{1}^{1} & \tilde{\beta}_{1}^{2} & \tilde{\beta}_{1}^{3} & \cdots & \tilde{\beta}_{1}^{m} \\
\tilde{\beta}_{2}^{1} & \tilde{\beta}_{2}^{2} & \tilde{\beta}_{2}^{3} & \cdots & \tilde{\beta}_{2}^{m} \\
\tilde{\beta}_{3}^{1} & \tilde{\beta}_{3}^{2} & \tilde{\beta}_{3}^{3} & \cdots & \widetilde{\beta}_{3}^{m} \\
\vdots & \vdots & \vdots & \ddots & \vdots \\
\tilde{\beta}_{n}^{1} & \tilde{\beta}_{n}^{2} & \tilde{\beta}_{n}^{3} & \cdots & \widetilde{\beta}_{n}^{m}
\end{array}\right],\right. \\
& \begin{array}{lllll}
E^{1} & E^{2} & E^{3} & \cdots & E^{m}
\end{array} \\
& I_{1}\left[\begin{array}{lllll}
\tilde{\beta}_{1}^{1} & \tilde{\beta}_{1}^{2} & \tilde{\beta}_{1}^{3} & \cdots & \tilde{\beta}_{1}^{m}
\end{array}\right] \\
& \begin{array}{llllll}
I_{2} & \tilde{\beta}_{2}^{1} & \tilde{\beta}_{2}^{2} & \tilde{\beta}_{2}^{3} & \cdots & \tilde{\beta}_{2}^{m}
\end{array} \\
& \widetilde{B}_{R}=I_{3} \mid \begin{array}{lllll}
\tilde{\beta}_{3}^{1} & \tilde{\beta}_{3}^{2} & \tilde{\beta}_{3}^{3} & \ldots & \widetilde{\beta}_{3}^{m}
\end{array}, \quad a=1,2, \ldots, m ; b=1,2, \ldots, n \text {, } \\
& I_{n}\left[\begin{array}{ccccc}
\vdots & \vdots & \vdots & \ddots & \vdots \\
\tilde{\beta}_{n}^{1} & \tilde{\beta}_{n}^{2} & \tilde{\beta}_{n}^{3} & \cdots & \tilde{\beta}_{n}^{m}
\end{array}\right] \\
& a=1,2, \ldots, m ; b=1,2, \ldots, n,
\end{aligned}
$$

where $n$ denotes the total number of items and $m$ denotes the total number of respondents and $\widetilde{\beta}_{b}^{a}=\left(l \beta_{b}^{a}, m \beta_{b}^{a}, u \beta_{b}^{a}\right)$ denotes the fuzzy implementation score of the items provided by the $a$ th respondent for the $b$ th item. After obtaining the three-dimensional assessment score $\left(\widetilde{B}_{A}, \widetilde{B}_{D}, \widetilde{B}_{R}\right)$, we calculate the average score $\widetilde{B}_{M}$ using

$$
\widetilde{B}_{M}=\frac{\widetilde{B}_{A}+\widetilde{B}_{D}+\widetilde{B}_{R}}{3},
$$

where $M$ represents mean, one example is given in Table 7 .

Step 2: As each expert judgment for the same items might be different according to their role, expertise, understanding, and perception of the subject matter. Therefore, we used the average score approach to get the cumulative fuzzy implementation score of each item by $m$ experts by equation (14):

$$
\widetilde{q}_{b}=\frac{1}{m}\left[\sum_{a=1}^{m} \widetilde{\beta}_{b}^{a}\right]
$$

where $\widetilde{q}_{b}=\left(l q_{b}, m q_{b}, u q_{b}\right)$ denotes the aggregate fuzzy importance of the $b$ th item.

Step 3. The accumulated TFN $\tilde{q}_{b}$ is obtained to calculate the BNP score. $\mathrm{BNP}_{\mathrm{Q}_{b}}$ can be obtained using equation (15):

$$
\mathrm{BNP}_{Q_{b}}=\frac{\left[\left(u q_{b}-l q_{b}\right)+\left(m q_{b}-l q_{b}\right)\right]}{3}+l q_{b},
$$

where $\mathrm{BNP}_{\mathrm{Q}_{b}}$ denotes the BNP value for the TFN $\widetilde{q}_{b}$ and $Q_{b}$ denotes the rating for the $b$ th item in the crisp number format.

Step 4. Since each item measures different aspects of the subcriteria, we calculate the average score to aggregate the best fuzzy importance $Q_{f}$ of each subcriterion (factor).

$$
Q_{f}=\frac{\sum_{b=1}^{k} Q_{b}}{k},
$$

where $Q_{b}$ represents the BNP implementation of the individual items in the corresponding factors (subcriteria), $k$ represents the total number of items in that factor while $\sum_{b=1}^{k} Q_{b}$ is the sum of the BNP weight of the all items in that factor.

Phase\#4: Evaluation via FMCDM. Once we came up with ranking and rating, then it is easy to estimate the possibility of success. The success value will be evaluated with predefined standard, i.e., $\geq 70 \%$ for adoption. The process is explained as follows.

3.3.7. Determining the Possibility of Successful Cloud Adoption for Software Testing. Once we came up with $R_{b}$ and $R_{f}$ the weights of the items and factors (subcriteria) and $Q_{b}$ and $Q_{f}$ the implementation of the items and factors in 


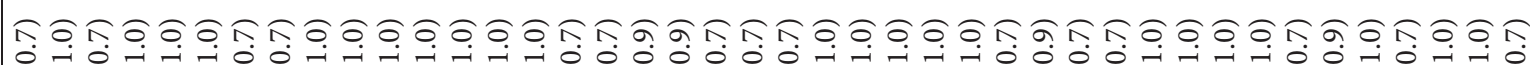
ڤึ

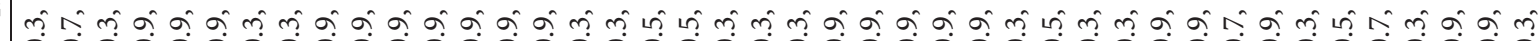

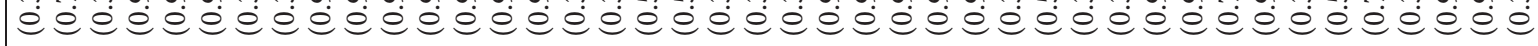

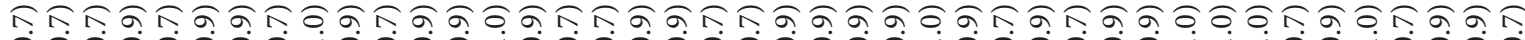

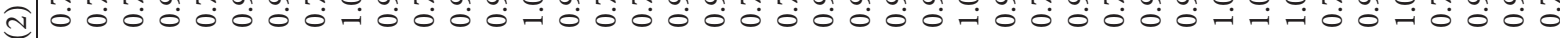

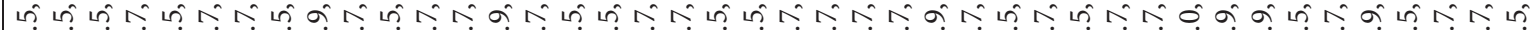

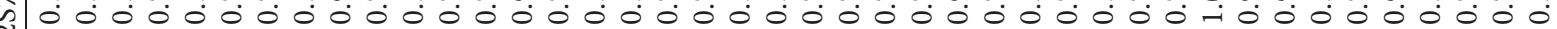

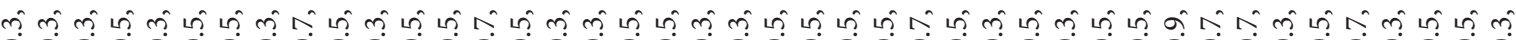

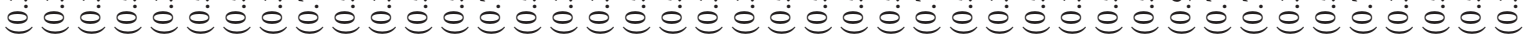

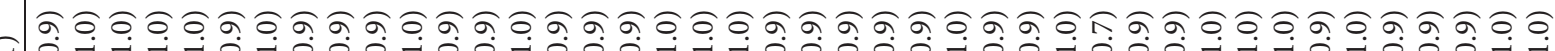

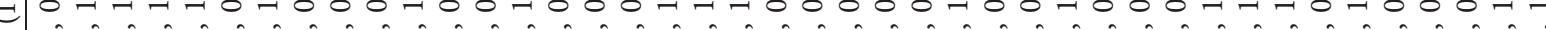

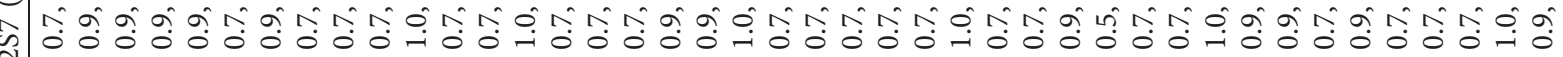

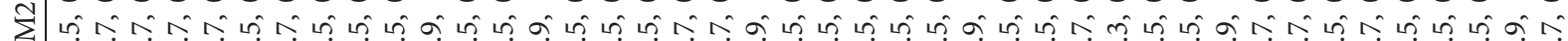

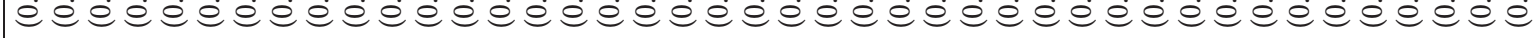

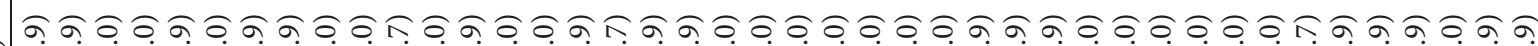

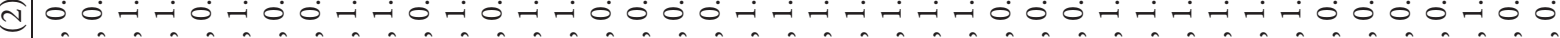

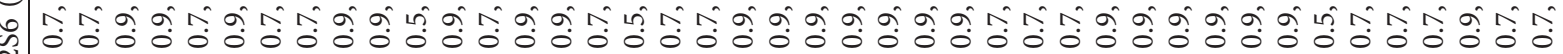

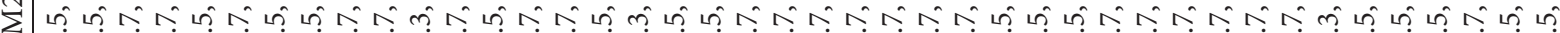

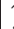

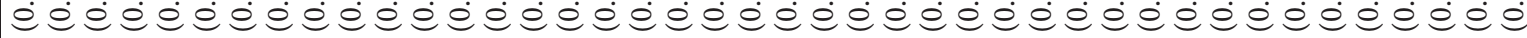

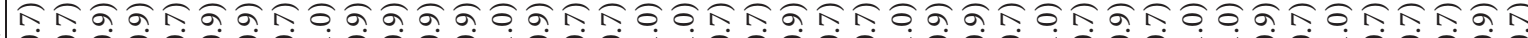

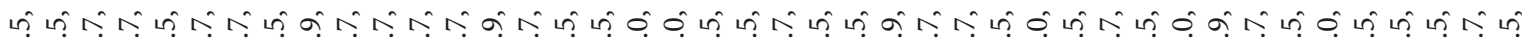

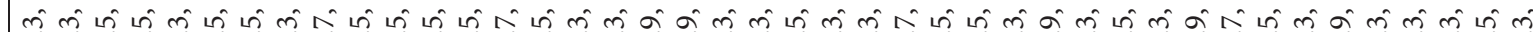
é é

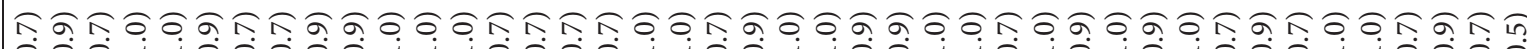

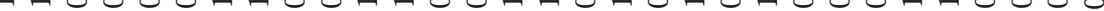

nी ñ 0000000000000000000000000000000000000000

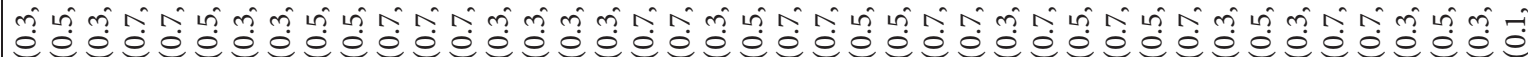

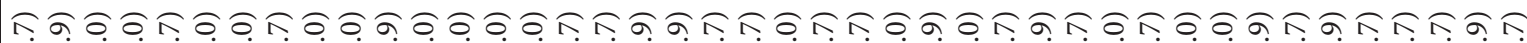

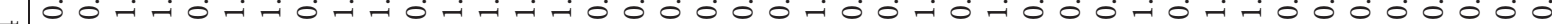

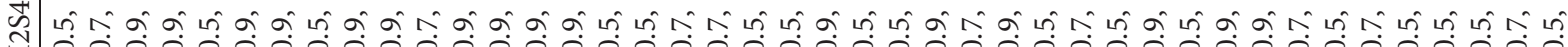

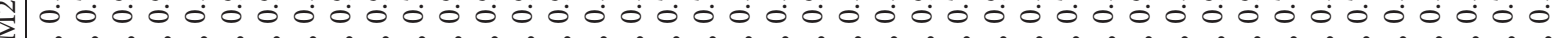

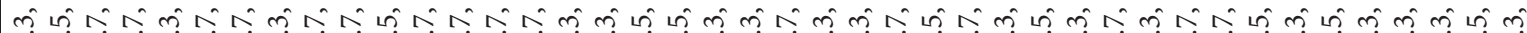

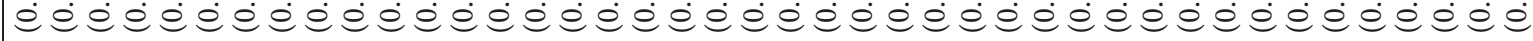

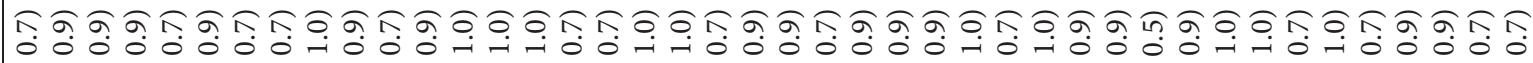

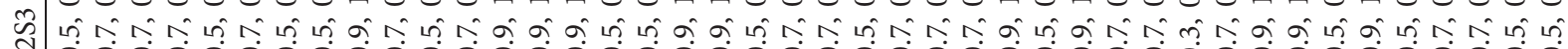

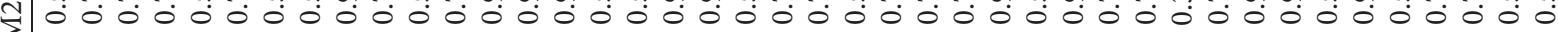

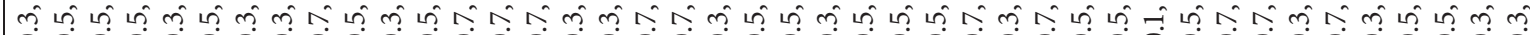

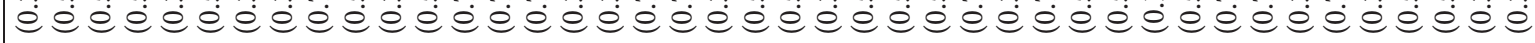

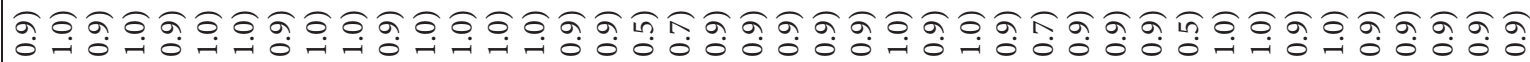

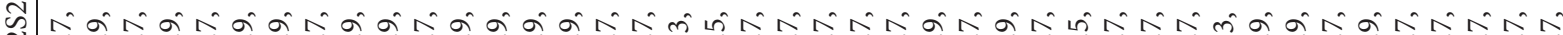

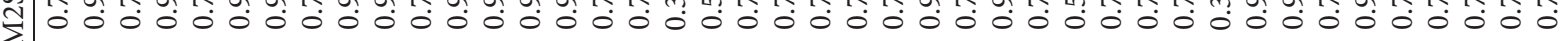

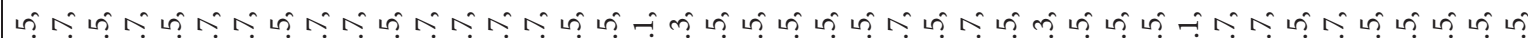

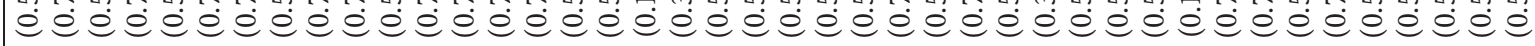

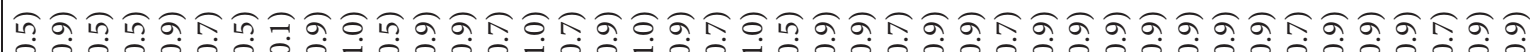

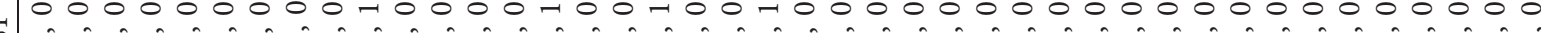

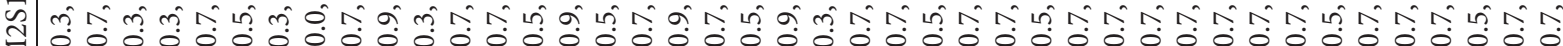
$\sum$ (n) हैं

芯

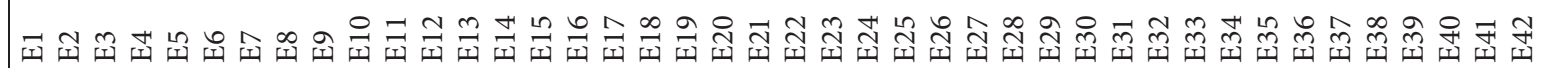




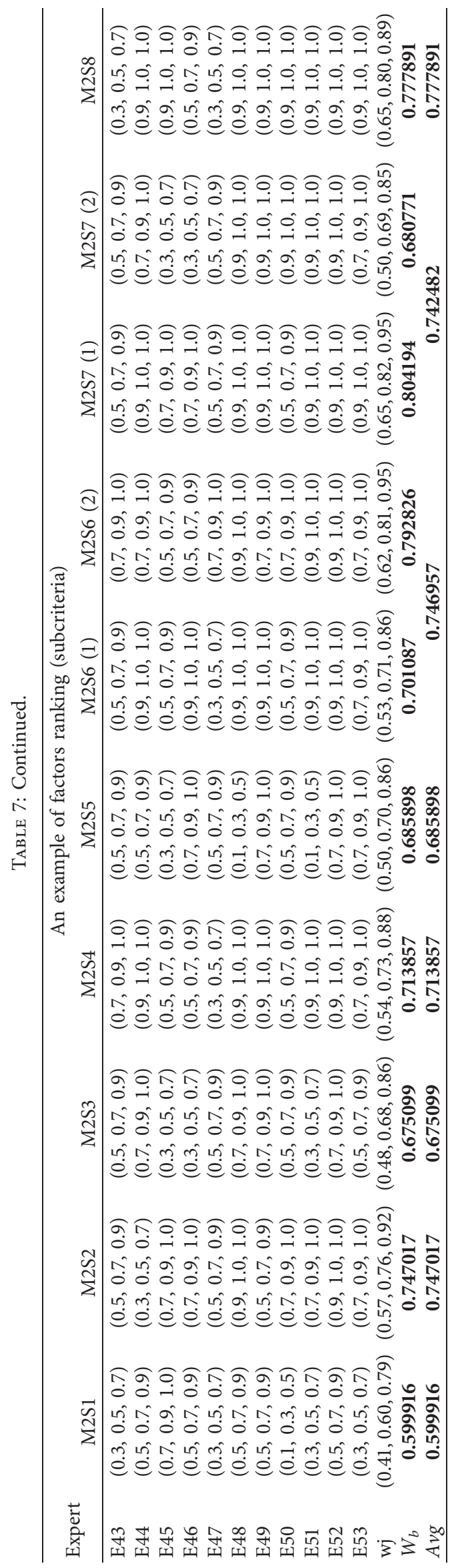


the respective organizations, then it is easy to estimate the possibility of success $P_{\text {sucess }}$ based on items using equation (17) as well as based on factors by equation (18)

$$
\begin{gathered}
P_{\text {sucess }}=R_{b} \times Q_{b}, \\
P_{\text {sucess }}=R_{f} \times Q_{f} .
\end{gathered}
$$

From the possibility of success, we can easily compute the possibility of failure.

Phase\#5: Assessment via FMCDM Using Motorola Assessment Tool. In the result of the evaluation, in case of a low outcome (less than 70\%), the model will pinpoint weak influential factors and predictors for further improvements while in case of high rate outcomes it will direct adoption and will signpost towards areas for enhancements.

\subsubsection{Identify the Weak Area for Future Enhancement.} The BNP weights of items are used to compute the BNP weights of factors and predictors. If $I_{f}$ represents the implementation of factor and $I_{p}$ represents the implementation of predictor, then it can be found using (19) and (20), respectively:

$$
I_{f}=\frac{Q_{f}}{k},
$$

where $k$ represents the total number of items in that factor $Q_{f}=\sum_{b=1}^{k} Q_{b}$ is the aggregate BNP implementation of all items implements that factor.

$$
I_{p}=\frac{Q_{p}}{h}
$$

where $h$ represents the total number of items in that predictor and $Q_{p}=\sum_{b=1}^{h} Q_{b}$ is the aggregate BNP implementation of all the items that measure that predictor.

\section{Results and Analysis}

This section demonstrates the outcomes of our study. This study proposes a technology acceptance model for CBT, based on the factors identified through the SLR and questionnaire survey, using the codified knowledge of experts and an intelligence technique for decision-making regarding CBT. The outcomes presented here are obtained based on the analysis of collected data in the first phase, i.e., ranking and rating of these data through the industrial survey and case study using FMCDM.

4.1. Results Obtained via Ranking the Criteria through an Empirical Survey. This section presents the results obtained by employing FMCDM techniques to analyze the data collected through SLR. We have identified 133 items to measure the ten predictors called the main criteria using 70 subfactors via FMCDM.

4.1.1. Weighs of the Factors (subcriteria) Using FMCDM. One hundred thirty-three items as shown in Appendix-A in Supplementary materials, were used in our scale to rank predictors and factors through a questionnaire survey. Ninety-five experts participate in the weighting process through a questionnaire survey. The weight calculation processes are as follows:

(1) These experts are asked to give their subjective judgment about the items using the linguistic scale presented in Table 1.

(2) The linguistic evaluations are then transcribed into the corresponding TFN as shown in Table 7 while taking predictor M2: "perceived performance expectancy" as an example.

(3) Because the perceptions of each expert are different due to their role, industrial experience, qualification, etc., equation (5) is used to get the synthesized aggregate TFN $\left(\widetilde{\omega}_{b}\right)$ as listed in Supplementary Table 2 column 4.

(4) Then, defuzzification of the TFN is carried out to obtain BNP in a crisp format using equation (6). The outcomes are shown in Supplementary Table 2 (BNP_WJ). The BNP value is used in the further calculation for global ranking $R_{b}$ and local ranking as shown in Supplementary Table 2 column 7.

(5) The crisp number obtained in step 4 is normalized and the normalized importance $R_{b}$ of items are obtained by using equation (7) which are further used to find an overall rank for each item. The outcomes are presented in Supplementary Table 2 column 8 (second last column). $R_{b}$ is used to obtain a global rank of each item as shown in Supplementary Table 2 column 9 (last column). As some factors and barriers are measured using more than one item, the individual item scores of a factor in BNF format are then converted to an average score in the same BNF format using equation (8) as shown in Supplementary Table 2 (last row). Referring to equation (8), the BNP weight of the factor (subcriteria) $W_{f}$ is an average of the BNP weight of all the items in that respective factor.

Using $W_{f}$, we obtained the local rank of the factors (subcriteria) within predictors (main criteria) as shown in Supplementary Table 2 second last column $\left(W_{f}\right)$.

4.1.2. Weights of the Predictor (Main Criteria) by FMCDM. Referring to equations (8) and (9), BNP weight of the factor (subcriteria), $W_{f}$ is the average of the BNP weight of all the items in that respective factor while the BNP weight of the predictor and $W_{p}$ is the sum of the BNP weights of all the items in that predictor. We first calculate $W_{f}$ by using equation (8), then we find the importance (rank) of factor within predictor (local weight) using $\left(W_{f}\right)$. The overall rank $R_{f}$ of a factor was obtained by dividing $W_{f}$ by sum of the weight of all factors, i.e., $\sum_{f=1}^{n} W_{f}$ refers to equation (10), which are 50.2752 in our study as given in Supplementary Table 2 (last row). For ranking predictors, we used the weight of the normalized importance of each predictor as calculated by equation (11). Ranks of the predictors are presented in 
Table 7, where $R_{p}$ is the normalized weight of the predictor and $W_{t}=\sum_{b=1}^{n} W_{b}$ shows the total weight, $W_{t}$ is the sum of the BNP weight $W_{b}$ of all the items and is accessible from Supplementary Table 2 (last row).

4.2. Results Obtained via Rating of the Influential Factors. To obtain the actual level of implementation of the influential factors using different items in the SDO organizations, a case study is carried out. The case study is a suitable method for providing enough evidence in the real-world industrial environment.

To be more certain and confident in our assessment, two distinct case studies were carried out at two distinct SDO companies. Companies are chosen because they provided particularly detailed descriptions of different SDO activities. If an item or factor has a strong implementation in the company, then the likelihood of success in the adoption of Cloud for future projects increases. The possible implementation of factor (subcriteria) concerning to each predictor (main-criteria) is calculated as follows:

Step 1: The participants in the case study were requested to provide their independent views about the extent of implementation of each item in their respective organizations from the three dimensions of Motorola by choosing linguistic terms shown in Supplementary Table 3 incorporating the Motorola guidelines given in Table 3 .

Step 2: The linguistic terms are then transcribed into corresponding TFN. An example of M2 (PPE): "perceived performance expectancy" are shown in Table 8.

Step 3: Three-dimensional scores in TFN format are then converted to an average score in the same TFN format using equation (13) as shown in Table 8.

Step 4: To aggregate the subjective judgments of the participants on the assessment of factors (because the perception of each expert is different due to their role, experiences, and education level, etc.). Equation (14) is used to get the synthesized TFN as listed in Table 8 (last row).

Step 5: Then defuzzification of the TFN is carried out to obtain BNP in the crisp format using equation (15) as shown in Supplementary Table 3.

4.3. Determining the Success Possibility of Cloud Adoption. Once we have an importance weight $R_{b}$ and possible implementation $Q_{j}$ of items, then it is easy to calculate the possibility of successful adoption using (19). The possibility of successful adoption for Company-A is shown in Table 9 (last column) while that of Company-B is shown in Table 10. The overall success value is equal to the sum of the success value of all items. Success value 0.5 indicates a $50 \%$ chance of both success and failure. Once we get success value, then the possibility of failure can be calculated using the common probability method.
4.4. Results Obtained through Evaluation of Predictors in the Case Organization. The implementation for company- $\mathrm{A}$ is given in Table 9. It is clear from Table 9 that company-A has not implemented any of the predictors (main-criteria). Furthermore, $P_{\text {Sucess }}$ is less than $70 \%$. Our model did not suggest company-A to adopt Cloud at this time because there is a $50 \%$ chance of both success and failure. To increase the chances of adoption, Company-A must focus on the perceived business concerns which are not implemented. Similarly, based on the results presented in Table 10 Company-B, has implemented seven predictors; however, $P_{\text {Sucess }}$ is still less than $70 \%$. Based on the implementation score, Company- $\mathrm{B}$ is relatively more secure if moving to the Cloud environment. For complete implementation, Company-B must focus on the weak factors under perceived business concerns (M10), which will further increase trust and will stimulate Company-B to adopt the Cloud.

\section{Summary and Discussion}

The objective of this study is to prioritize the predictors as well as to rank the overall subcriteria. Firstly, the ten main criteria are ranked as CC adoption "Feasibility planning and risk analysis, 0.11"; "Perceived degree of Cloud resource utilization, 0.1168"; "Perceived effort expectancy, 0.1141"; "Organization competency and capacity, 0.1128"; "Perceived degree of trust, 0.111"; "Perceived business concerns, 0.1020"; "Organizational dynamics and business drivers, 0.0999"; "External stimuli, 0.0924"; "Perceived performance expectancy, 0.0745"; and "Perceived economic benefits of Cloud adoption, 0.057."

According to the rank of predictors, we find that feasibility planning and risk analysis, perceived degree of Cloud resource utilization, and perceived effort expectancy are the top three predictors of Cloud adoption in the context of software testing. It means, currently testing in the Cloud does not apply to all types of software. Furthermore, most of the testing techniques cannot be applied in the Cloud environment. Additionally, currently testing in the Cloud is not fully automated and testers are left with the laborious tasks of writing scripts and configuring the test environment. Utilization of Cloud resources is also limited due to the viability and suitability of different types of software to be tested and types of tests performed in the Cloud environment. Furthermore, human testers are still needed to conduct testing. This might be the reason that "perceived economic" benefit of Cloud adoption has low weight and ranks low. Besides, perceived performance expectancy is the second least significant predictor in our study. This might be the reason that the adoption of CC in the domain of software testing is very slow compared with that in other fields. The following studies support the results:

(i) The first step in moving to the Cloud is the deep understanding of CC resources and how they relate to their needs. It is also imperative that SDO realizes and analyzes the CC value-added features and goes on only if there is a growing need for this approach [56]. 


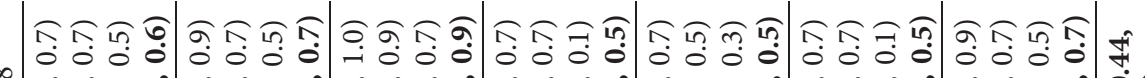

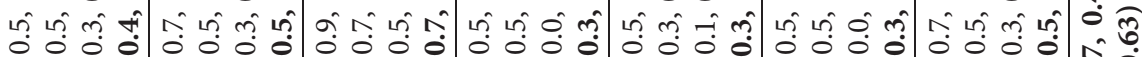
ली

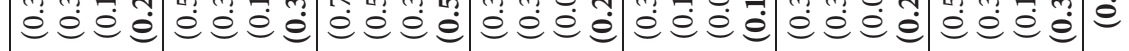

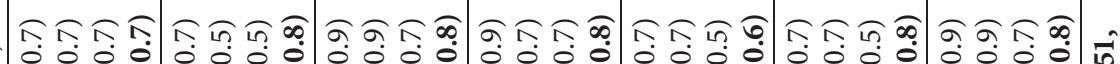
I

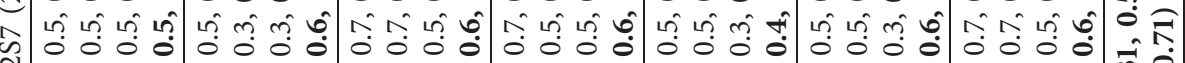

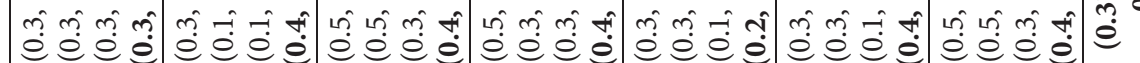

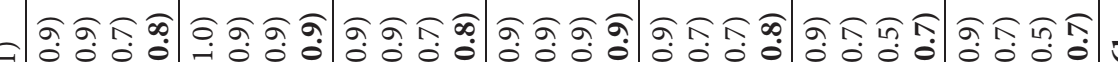

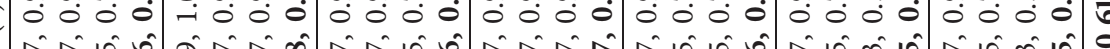

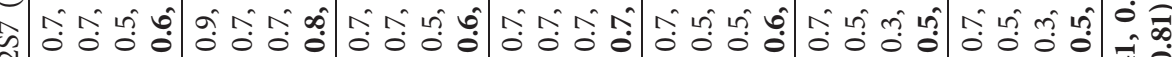

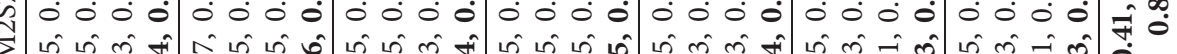

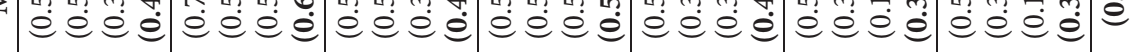

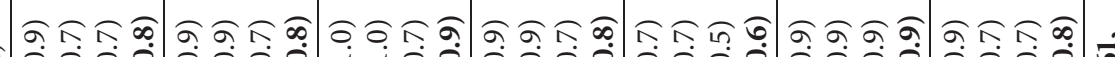
(

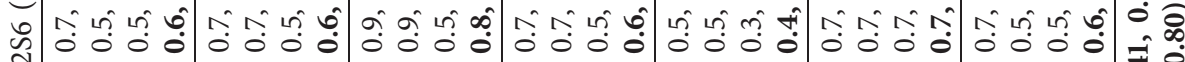

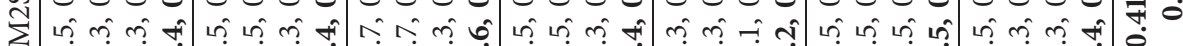

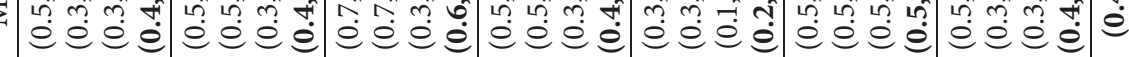

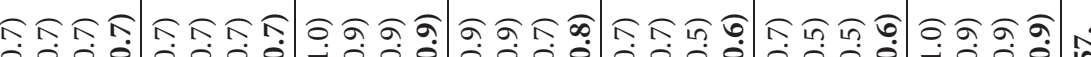

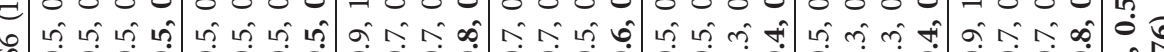

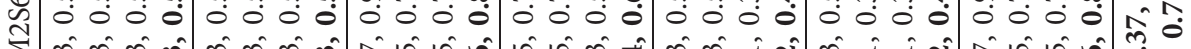

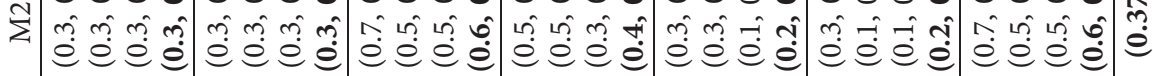

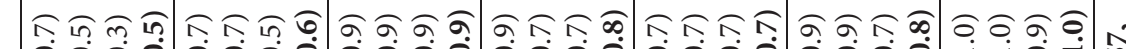

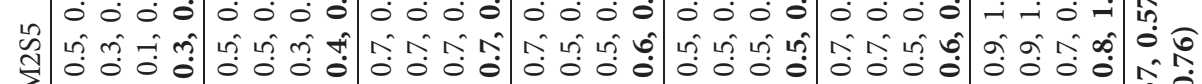
$\Sigma$ री

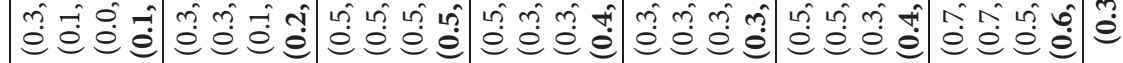

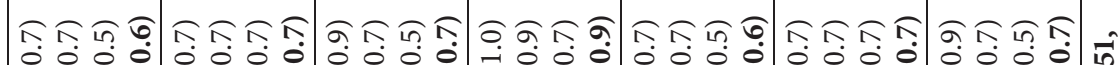
苞 告

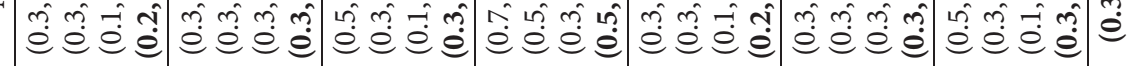

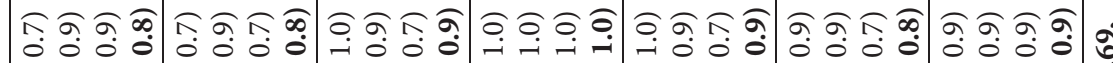

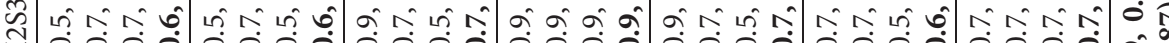

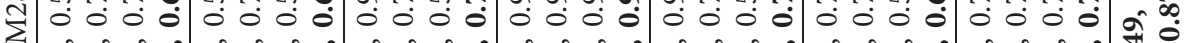

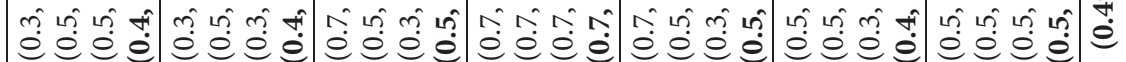

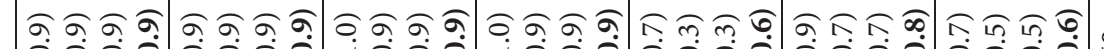

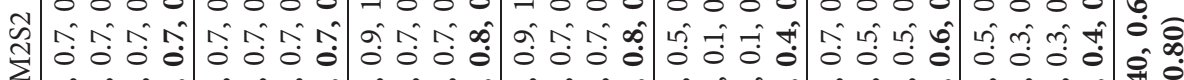

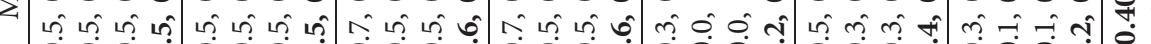

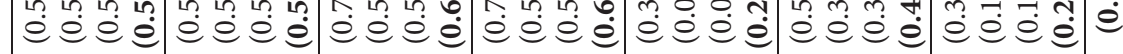

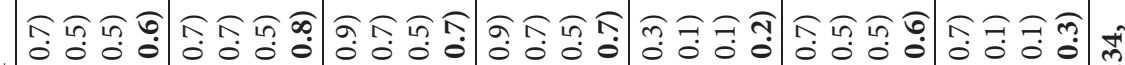

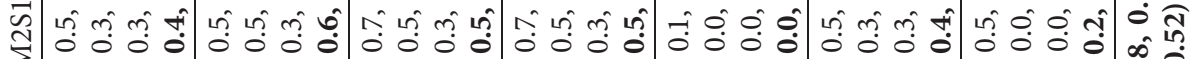

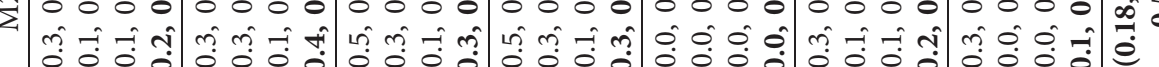

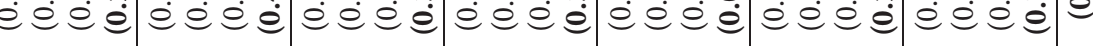


(ii) To find an answer to the question "when to migrate testing to the CC platform?" one must check the characteristics of SUT, perceived support of the testing methods in the CC environment, preferences, nature, and dependency of test execution [74]. If the majority of these subfactors cannot operate in the Cloud environment, then Cloud adoption is considered feasible; otherwise, a decision to stop the migration can be made [74].

(iii) Before going to adopt the Cloud testing service, you have to look at ways through which testing is conducted and testing services the service providers offer, in order to plan best to maximum utilize Cloud platform for their testing [25].

(iv) TaaS can be effusively embraced by the industry if it supports more types of testing such as agile development via continuous regression testing [56].

(v) Perceived ease of use contributes significantly towards a willingness to adopt CC services [45]. The outcomes show that convenience and ease of use are the key motivators for Cloud services adoption [45].

(vi) Automation could encourage the adoption of a service. On the contrary, if a service requires tremendous human effort, this might negatively affect adoption [75].

(vii) Despite the great importance of Cloud-based testing, it is extremely complex and at present lacks proper tools to support it [76].

(viii) Extra efforts are required to deploy application and setup test environment in the Cloud [77].

(ix) For simple test cases, the use of CC might increase the task cost and system complexity [78].

(x) Automation encourages the adoption of the CBT technique. On the contrary, if a tool or service requires huge efforts, this might limit its adoption [75].

(xi) To improve quality and reduce the cost of test automation is the most important means [79].

(xii) According to a topical survey [80], presently $28 \%$ of test cases are automated whereas the executives demand to raise this amount shortly. Employing test automation mainstream in the software industry, more and more companies will adopt CBT.

(xiii) The authors of [56] suggest that future work on TaaS needs to focus on entirely automatic test cases. They examine the currently available tools and services and find that only BlazeMeter can offer fully automated performance and load testing [56].

The ranks of the subcriteria in connection to "CC adoption" for software testing are given in Supplementary Table 2. Among the 70 subcriteria, top ten criteria are support for multidirectional testing, 0.018547; top management support, 0.018471; interface usability, 0.017637; standardization; 0.016031; reduced employee cost, 0.017257; pricing and deployment models, 0.016830; employee support for CBT, 0.016723; resources availability, 0.016637; perceived IT risks, 0.013693; and service automation, 0.016500 .

Ten least significant factors in decreasing order of weights from 61 to 70 are regulation requirements, 0.012336 ; improve developer-tester collaboration, 0.011930; risk analysis, 0.011784; competitor's pressure, 0.011492 ; security, 0.011027; incompatibility, 0.008024 ; performance uncertainty and outage, 0.007983; legal concerns, 0.005898; insufficient QoS guarantee via SLA, 0.004413; and concerns over confidentiality, 0.004020 .

Priyadarshinee et al. [71] rank the factors through fuzzy AHP. They identify organizational risks, sharing and collaboration, confidentiality and integrity, and security and privacy as top factors. According to them, security and privacy, sharing and collaboration, confidentiality, and integrity are the top four factors that have a major effect on the adoption of CC. Competitive pressure is ranked in the middle by us (and it is ranked 20th out of 51 by them), but unlike them, we have relatively low weight for security and privacy. The reason might be that testing data is not sensitive; therefore, organizations are not worried about the security of data.

(i) Migrating the testing to the CC environment is anticipated to be a safe decision because the testing will not contain any sensitive business data [2].

(ii) Migration will have a nominal impact on corporate work as usual deeds [26].

(iii) For organizations having no concerns for security, software testing can be one of the suitable areas that can be moved to the Cloud platform [49].

However, the privacy of the personal information and test results still concerns to SDO. Assurance of privacy, disaster recovery, and back up inspire the confidence of the client in Cloud adoption for software testing.

(i) Since testing does not contain sensitive information, the security concerns are related to the privacy of resources and confidentiality of test results [81].

(ii) Before adopting Cloud for software testing, one should decide whether the Cloud is safe for their sensitive information [35].

(iii) An acceptable testing service essentially addresses concerns related to multitenancy as well as to the safety of uploaded material and confidentiality of test results [82].

(iv) All tenants' data in Cloud are stored in a shared infrastructure using a shared single instance, hence making it difficult to ensure data confidentiality [83].

To assess our proposed framework, we have executed two case studies in the SDO planning to adopt Cloud for software testing shortly. At the end of the case study, a focus group session was conducted among the participants to 
TABLE 9: Evaluation of predictor in case organization (Company-A).

\begin{tabular}{|c|c|c|c|c|}
\hline Name of predictor & $Q_{p}$ & \# items & $I_{p}$ & $P_{\text {Success }}$ \\
\hline M01: perceived economic benefits of Cloud adoption & 4.7540 & 9 & 0.5282 & \multirow{10}{*}{50.3122 approx. $50.3 \%$} \\
\hline M02: perceived performance expectancy & 5.4460 & 10 & 0.5446 & \\
\hline M03: perceived effort expectancy & 8.1333 & 14 & 0.5810 & \\
\hline M04: perceived degree of Cloud resource utilization & 8.6905 & 14 & 0.6208 & \\
\hline M05: organizational dynamics and business drivers & 7.4556 & 12 & 0.6213 & \\
\hline M06: perceived degree of trust & 8.2571 & 16 & 0.5161 & \\
\hline M07: external stimuli (pressure + support) & 5.0429 & 12 & 0.4202 & \\
\hline M08: organizational competency and readiness & 8.3413 & 13 & 0.6416 & \\
\hline M09: feasibility planning and risk analysis & 9.8943 & 16 & 0.6184 & \\
\hline M10: perceived business concerns & 8.0921 & 18 & 0.4496 & \\
\hline
\end{tabular}

TABLE 10: Evaluation of predictor in case organization (Company-B).

\begin{tabular}{|c|c|c|c|c|}
\hline Name of predictor & $Q_{p}$ & \# items & $I_{p}$ & $P_{\text {Success }}$ \\
\hline M01: perceived economic benefits of Cloud adoption & 6.174 & 09 & 0.6860 & \multirow{10}{*}{61.26407 approx. $61.3 \%$} \\
\hline M02: perceived performance expectancy & 6.996 & 10 & 0.6996 & \\
\hline M03: perceived effort expectancy & 10.031 & 14 & 0.7165 & \\
\hline M04: perceived degree of Cloud resource utilization & 10.325 & 14 & 0.7375 & \\
\hline M05: organizational dynamics and business drivers & 9.4556 & 12 & 0.7880 & \\
\hline M06: perceived degree of trust & 8.2571 & 16 & 0.5161 & \\
\hline M07: external stimuli (pressure + support) & 7.0429 & 12 & 0.5869 & \\
\hline M08: organizational competency and readiness & 9.1930 & 13 & 0.7072 & \\
\hline M09: feasibility planning and risk analysis & 11.843 & 16 & 0.7402 & \\
\hline M10: perceived business concerns & 10.921 & 18 & 0.6067 & \\
\hline
\end{tabular}

obtain feedback and the model was revised accordingly. Case study method proves the most powerful instrument for validation in empirical software engineering [72].

In this study, the Motorola assessment instrument and the TFN scale are used in a fuzzy environment. Our contribution is the introduction of TFN scores and the updated guidelines for a 7-point Likert scale. The existing Motorola guidelines are suitable for a 5-point Likert scale only; we have added two more rows, one at the start and the other at the end to make it convenient for measurement on a 7-point Likert scale. The same instrument is also used for the evaluation of SOVRM [84], SPIIMM [354], and SOPM [20]. This tool has been used in CMMI to evaluate the organizations' present position comparable to the CMM and to recognize weak areas that require improvements and management attention [73].

Evaluation results regarding factors using the Motorola assessment instrument are presented in Table 3. According to Motorola assessments, factors having an average evaluation or implementation score greater than 0.7 will be considered implemented while scores less than 0.7 will be marked as weakness. For instance, in predictor perceived economic benefits (M1), two factors such as market place establishment (MIS6) and access to global markets (M1S7) are not implemented properly. The reason might be that Company-A, which does not want these two goals to be achieved through CC adoption.

(i) The biggest challenge is to ensure global availability all the time [56].

(ii) Currently, there is no comprehensive model to test the mobile CC applications. Furthermore, they state that the new Cloud testing methods are restricted to the applications with special features and can only support limited types of tests [85].

All factors in perceived performance expectancy (M2) have scores less than 0.7 except better service availability and reliability (M2S3). It means Company-A is optimistic only about Cloud reliability and availability. It can be observed from Table 3 that "improves developer-tester collaboration" (M2S1) gets a very low implementation score, i.e., lower than $35 \%$. It means regarding Company-A, adoption of CC will not improve developer and tester communication to a high level. Additionally, "better usability and easy deployment" gets a score (56\%) which is found very high in the other Cloud adoption study such as $[45,86]$. The reason might be that as compared with other Cloud services, testing is not fully automated. The following literature proves the claim:

(i) CC adoption is neither feasible and risk-free all the time nor an automatic or easy process [13].

(ii) The use of CC might increase the task cost and system complexity in case of simple tests [78].

(iii) According to a well-established French testing company called Sogeti, merely $28 \%$ of the test cases are presently automated, whereas the executives demand to raise this amount soon. Utilizing test automation in the software industry more and more companies will adopt CBT [80].

(iv) Future work on TaaS needs to focus on entirely automatic test cases. They have examined the currently available tools and services and found that 
only BlazeMeter can offer fully automated performance and load testing [56].

In connection to the perceived degree of Cloud resource utilization (M4), we got a relatively low implementation of factors "varieties of service utilization" (M4S3), "support for Global software development" (M4S5), and "support for ubiquity and mobile testing" (M4S6).

(i) Not all types of testing are suitable to be performed in the Cloud. For instance, high-volume automated testing, unit testing (particularly large regression suites), and performance testing are ideal choices to be migrated to the Cloud environment [14].

(ii) TaaS can be effusively embraced by the industry if it supports more types of testing such as agile development via continuous regression testing [56].

(iii) The biggest challenge is to ensure global availability all the time [56].

(iv) Currently, there is no comprehensive model to test the Cloud mobile applications. Furthermore, they state that the new Cloud testing methods are restricted to applications with special features and can only be used in some types of tests [85].

\section{Conclusion}

This study proposes that software testing should be moved to the Cloud. Additionally, this study explores the determinants and predictors of Cloud adoption for software testing. For this purpose, this study explores the technical aspect of software testing in the Cloud through the FMCDM approach and further develops a technology acceptance model. An expert system based on the codified knowledge of an expert and artificial intelligence techniques such as MCDM is applied to help organizations decide Cloud adoption for software testing. The proposed model includes various predictors and determinants that will guide software development organizations (SDO) towards Cloud adoption for software testing. The model is developed using a five-phase analytic approach. Data are collected via SLR and questionnaire-based survey while analysis is made through fuzzy multicriteria decisionmaking (FMCDM) approach.

The results of this study suggest that Cloud service providers need to provide support to SDO who are presently working in the CBT domain, develop a cooperative environment for the CC adoption, and eliminate any ambiguity regarding this technology. Besides, the CC providers should clarify the advantages of Cloud services over in-house services. Furthermore, they must focus on concerns such as locality and privacy faced by the SDO and resolve those problems by forming an unrelenting relationship with the clients. The Cloud providers have to assure the SDO that their testing data are safe and accessible upon request around the clock. They should increase their reliability to get confidence, faith, and trust of the SDO about their services. A trial chance may be provided to the SDO to try the Cloud services out before its actual implementation. The provider government has to play a significant role in building trust regarding government intrusion among the SDO. The Client needs to implement CBT and it is very off-beamed to think that $\mathrm{CBT}$ is meant for larger companies only.

\section{Threats to Validity}

The empirical survey was based on the results of SLR; this two-phase framework ensures content validity. Construct validity is concerned with measurement scale whether the measurement scales represent the attributes being measured. The attributes of this research study were taken from a considerable amount of previous research $[25,42-48]$ and experiencing a systematic literature review $[20,87,88]$. The respondents of the survey confirm the relevance of the attributes selected. Further, the inner reliability of survey responses was assessed using Cronbach's alpha coefficient, which is $0.89(>0.70)$, which demonstrates the reliability of data and scale. To internal validity, the SLR findings were used as input for the design of the questionnaire.

The empirical study part of this research engaged participants mainly from the Asian countries only. However, to lessen population prejudice, contributors from other countries such as North America were also invited to include diverse perspectives. Fifty experts voluntarily participated in this exploratory study and there were no previous bonds between the participants and researchers. Contributors were informed that their participation is entirely voluntary and they can withdraw at any time during any stage of the survey if they want. However, to ensure external validity and to diminish any possible bias, the ninety-five contributors were chosen from 20 different countries. Besides, most of the participants had worked in a range of small, medium, and large multinational organizations. Although we cannot claim that all the contributors from these 20 countries would agree with us, we believe that they provide a descriptive sample. In an empirical survey-based research, it is hard to obtain a fully representative sample and to deal with them in an entirely objective fashion [89]. To overcome these limitations, only those participants were included who are involved in test automation. The claim/ relevant expertise of the participants were verified by inculcating some open-ended questions in the questionnaire, which were difficult to answer by an ordinary tester or manager etc. This situation might create difficulties when contributors' judgments may be inaccurate or factors supposed to have a significant interrelation for adoption may not be significant at all. However, similar to other opinion-based empirical research studies [25, 43-48], we have full confidence that the findings of this research are based on the data that have been collected from the relevant participants who have been involved and have vastly diversified experience in SDO.

\section{Data Availability}

The data used to support the study are provided in the manuscript. 


\section{Conflicts of Interest}

The authors declare that they have no conflicts of interest.

\section{Acknowledgments}

This work was supported by National Key Research and Development Program of China (No. 2018YFB1003800), National Natural Science Foundation of China (No. 61972414), Beijing Natural Science Foundation (No. 4202066), and Fundamental Research Funds for Central Universities (Nos. 2462020YJRC001 and 2462018YJRC040).

\section{Supplementary Materials}

All the necessary data are provided in Supplementary Tables 1 to 3. (Supplementary Materials)

\section{References}

[1] M. Madan, M. Dave, and A. Tandon, "Challenges in testing of cloud based application," International Journal of Advanced Research in Computer Science and Electronics Engineering, vol. 5, no. 1, pp. 28-31, 2016.

[2] M. S. N. Murthy and V. Suma, "Software testing and its scope in CLOUD: a detailed survey," in Proceedings of the 2017 International Conference on Innovative Mechanisms for Industry Applications (ICIMIA), pp. 269-273, Bengaluru, India, February 2017.

[3] O. Taipale, J. Kasurinen, K. Karhu, and K. Smolander, "Tradeoff between automated and manual software testing," International Journal of System Assurance Engineering and Management, vol. 2, no. 2, pp. 114-125, 2011.

[4] V. Garousi, M. V. Mäntylä, A. Betin-Can, and S. Mirshokraie, "When and what to automate in software testing? A multivocal literature review," Information and Software Technology, vol. 76, pp. 92-117, 2016.

[5] G. N. Iyer, J. Pasimuthu, and R. Loganathan, "PCTF: an integrated, extensible cloud test framework for testing cloud platforms and applications," in Proceedings of the 13th International Conference on Quality Software (ICSQ), pp. 135138, Dallas, TX, USA, May 2013.

[6] S. Marston, Z. Li, S. Bandyopadhyay, and A. Ghalsasi, "Cloud computing-the business perspective," in Proceedings of the 44th Hawaii International Conference on System Sciences, pp. 1-11, Kauai, HI, USA, January 2011.

[7] M. Armbrust, I. Stoica, M. Zaharia et al., "A view of cloud computing," Communications of the ACM, vol. 53, no. 4, pp. 50-58, 2010.

[8] J. Luftman, H. S. Zadeh, B. Derksen, M. Santana, E. H. Rigoni, and Z. Huang, "Key information technology and management issues 2011-2012: an international study," Journal of Information Technology, vol. 27, no. 3, pp. 198-212, 2012.

[9] L. A. Kappelman, E. R. Mclean, J. N. Luftman, and V. Johnson, "Key issues of IT organizations and their leadership: the 2013 SIM IT trends study," Mis Quarterly Executive, vol. 12, no. 4, p. 7, 2013.

[10] A. Elumalai, I. Starikova, and S. Tandon, IT as a Service: From Build to Consume, McKinsey and Company, New York, NY, USA, 2018.

[11] J. Avrane-Chopard, T. Bourgault, A. Dubey, and L. Moodley, "Big business in small business: cloud services for SMBs,"
Telecommunications, Media, and Technology Practice, McKinsey and Company, New York, NY, USA, 2014.

[12] J. Gao, X. Bai, W. Tsai, and T. Uehara, "Testing as a service (TaaS) on clouds,", in Proceedings of the IEEE Seventh International Symposium on Service-Oriented System Engineering (SOSE), pp. 212-223, San Francisco Bay, CA, USA, March 2013.

[13] H. Srikanth, M. Cashman, and M. B. Cohen, "Test case prioritization of build acceptance tests for an enterprise cloud application: an industrial case study," Journal of Systems and Software, vol. 119, pp. 122-135, 2016.

[14] T. Parveen and S. Tilley, "When to migrate software testing to the cloud?" in Proceedings of the Third International Conference on Software Testing, Verification, and Validation Workshops (ICSTW), pp. 424-427, Paris, France, April 2010.

[15] Z. Ganon and I. E. Zilbershtein, "Cloud-based performance testing of network management systems," in Proceedings of the 14th IEEE International Workshop on Computer Aided Modeling and Design of Communication Links and Networks (CAMAD), pp. 1-6, Pisa, Italy, June 2009.

[16] L. Riungu-Kalliosaari, "Empirical study on the adoption, use and effects of cloud-based testing," Doctoral Dissertation, Lappeenranta University of Technology, Lappeenranta, Finland, 2014.

[17] D. Kumar and K. K. Mishra, "The impacts of test automation on software's cost, quality and time to market," Procedia Computer Science, vol. 79, pp. 8-15, 2016.

[18] S. Ali and N. Ullah, "Critical influential factors for software testing-as-a-service adoption: preliminary findings from systematic literature review," in Proceedings of the International Conference on Electrical, Communication, and Computer Engineering (ICECCE), pp. 1-6, Swat, Pakistan, July 2019.

[19] M. Diaz and J. Sligo, "How software process improvement helped Motorola," IEEE Software, vol. 14, no. 5, pp. 75-81, 1997.

[20] S. Ali and S. U. Khan, "Software outsourcing partnership model: an evaluation framework for vendor organizations," Journal of Systems and Software, vol. 117, pp. 402-425, 2016.

[21] S. Ali, H. Li, S. U. Khan, Y. Zhao, and L. Li, "Fuzzy multi attribute assessment model for software outsourcing partnership formation," IEEE Access, vol. 6, pp. 55431-55461, 2018.

[22] S. Ali and H. Li, "Moving software testing to the cloud: an adoption assessment model based on fuzzy multi-attribute decision making algorithm," in Proceedings of the IEEE 6th International Conference on Industrial Engineering and Applications (ICIEA), pp. 382-386, Tokyo, Japan, April 2019.

[23] R. A. Khan, M. Y. Idris, S. U. Khan et al., "An evaluation framework for communication and coordination processes in offshore software development outsourcing relationship: using fuzzy methods," IEEE Access, vol. 7, pp. 112879-112906, 2019.

[24] X. Bai, M. Li, B. Chen, W. T. Tsai, and J. Gao, "Cloud testing tools," in Proceedings of the IEEE 6th International Symposium on Service Oriented System Engineering (SOSE), pp. 1-12, Irvine, CA, USA, December 2011.

[25] L. Riungu-Kalliosaari, O. Taipale, K. Smolander, and I. Richardson, "Adoption and use of cloud-based testing in practice," Software Quality Journal, vol. 24, no. 2, pp. 337-364, 2016.

[26] I. C. Priyanka, I. Chana, and A. Rana, "Empirical evaluation of cloud-based testing techniques," ACM Sigsoft Software Engineering Notes, vol. 37, no. 3, pp. 1-9, 2012. 
[27] C. Jia, Y. Cai, Y. T. Yu, and T. H. Tse, “ $5 \mathrm{~W}+1 \mathrm{H}$ pattern: a perspective of systematic mapping studies and a case study on cloud software testing," Journal of Systems and Software, vol. 116, pp. 206-219, 2016.

[28] W.-T. Tsai and G. Qi, "Integrated fault detection and test algebra for combinatorial testing in TaaS (Testing-as-a-Service)," Simulation Modelling Practice and Theory, vol. 68, pp. 108-124, 2016.

[29] S. Zein, N. Salleh, and J. Grundy, "A systematic mapping study of mobile application testing techniques," Journal of Systems and Software, vol. 117, pp. 334-356, 2016.

[30] T. Banzai, H. Koizumi, R. Kanbayashi et al., "Design of a software testing environment for reliable distributed systems using cloud computing technology," in Proceedings of the IEEE/ACM International Symposium Cluster Computing and the Grid (CCGRID), pp. 631-636, Melbourne, Australia, May 2010.

[31] R. M. Ashfaq, "A new testing framework for cloud-based application," Journal of Software Engineering \& Intelligent Systems, vol. 2, no. 3, pp. 157-163, 2017.

[32] P. Jamshidi, A. Ahmad, and C. Pahl, "Cloud migration research: a systematic review," IEEE Transactions on Cloud Computing, vol. 1, no. 2, pp. 142-157, 2013.

[33] P. Harikrishna and A. Amuthan, "A survey of testing as a service in cloud computing," in Proceedings of the International Conference on Computer Communication and Informatics (ICCCI), pp. 1-5, Coimbatore, India, 2016.

[34] A. A. Ahmad, P. Brereton, and P. Andras, "A systematic mapping study of empirical studies on software cloud testing methods," in Proceedings of the IEEE International Conference on Software Quality, Reliability and Security Companion (QRS-C), pp. 555-562, Prague, Czech Republic, July 2017.

[35] A. F. Mohammad and H. McHeick, "Cloud services testing: an understanding," Procedia Computer Science, vol. 5, pp. 513520, 2011.

[36] J. Fernandes and F. Gemmer, "Application testing on the cloud: smart testing for agile enterprises," 2020, https://www. oracle.com/technetwork/oem/s317292-app-testing-in-cloud181639.pdf.

[37] A. Bertolino, L. Nautiyal, and Preeti, "Annotated buzzwords and key references for software testing in the cloud," in Proceedings of the International Conference on Computing, Communication and Automation (ICCCA), pp. 893-900, Noida, India, May 2017.

[38] S. Nachiyappan and S. Justus, "Cloud testing tools and its challenges: a comparative study," Procedia Computer Science, vol. 50, pp. 482-489, 2015.

[39] M. Tiitinen, "Key factors for selecting software testing tools," Master Degree in Business Informatics Master Business Informatics, Helsinki Metropolia University of Applied Sciences, Helsinki, Finland, 2013.

[40] M. S. N. Murthy and V. Suma, "A study on cloud computing testing tools," in ICT and Critical Infrastructure: In Proceedings of the 48th Annual Convention of Computer Society of India, vol. I, pp. 605-612, Springer, Cham, Switzerland, 2014.

[41] M. S. Geetha Devasena, V. Krishna Kumar, and R. Kingsy Grace, "LTTC: a load testing tool for cloud," in Proceedings of International Conference on Communication and Networks, pp. 689-698, Singapore, 2017.

[42] M. O. Alassafi, A. Alharthi, R. J. Walters, and G. B. Wills, "A framework for critical security factors that influence the decision of cloud adoption by Saudi government agencies," Telematics and Informatics, vol. 34, no. 7, pp. 996-1010, 2017.
[43] N. Alkhater, R. Walters, and G. Wills, "An empirical study of factors influencing cloud adoption among private sector organizations," Telematics and Informatics, vol. 35, no. 1, 2018.

[44] Y. Alshamaila, S. Papagiannidis, and F. Li, "Cloud computing adoption by SMEs in the north east of England," Journal of Enterprise Information Management, vol. 26, no. 3, pp. 250275, 2013.

[45] P. Gupta, A. Seetharaman, and J. R. Raj, "The usage and adoption of cloud computing by small and medium businesses," International Journal of Information Management, vol. 33, no. 5, pp. 861-874, 2013.

[46] P.-F. Hsu, S. Ray, and Y.-Y. Li-Hsieh, "Examining cloud computing adoption intention, pricing mechanism, and deployment model," International Journal of Information Management, vol. 34, no. 4, pp. 474-488, 2014.

[47] S. K. Sharma, A. H. Al-Badi, S. M. Govindaluri, and M. H. AlKharusi, "Predicting motivators of cloud computing adoption: a developing country perspective," Computers in Human Behavior, vol. 62, pp. 61-69, 2016.

[48] R. D. Raut, B. B. Gardas, M. K. Jha, and P. Priyadarshinee, "Examining the critical success factors of cloud computing adoption in the MSMEs by using ISM model," The Journal of High Technology Management Research, vol. 28, no. 2, pp. 125-141, 2017.

[49] K. Incki, B. Ari, and H. Sozer, "A survey of software testing in the cloud," in Proceedings of the IEEE Sixth International Conference on Software Security and Reliability (SESR), pp. 18-23, Gaithersburg, MD, USA, August 2012.

[50] R. R. Kumar and C. Kumar, "A multi criteria decision making method for cloud service selection and ranking," International Journal of Ambient Computing and Intelligence, vol. 9, no. 3, pp. 1-14, 2018.

[51] P. Priyadarshinee, R. D. Raut, M. K. Jha, and B. B. Gardas, "Understanding and predicting the determinants of cloud computing adoption: a two staged hybrid SEM - neural networks approach," Computers in Human Behavior, vol. 76, pp. 341-362, 2017.

[52] S. Nazir, S. Shahzad, M. Nazir, and H. U. Rehman, "Evaluating security of software components using analytic network process," in Proceedings of the 11th International Conference on Frontiers of Information Technology (FIT), pp. 183-188, Islamabad, Pakistan, December 2013.

[53] S. Nazir, S. Anwar, S. A. Khan et al., "Software component selection based on quality criteria using the analytic network process," Abstract and Applied Analysis, vol. 2014, Article ID 535970, 12 pages, 2014.

[54] S. Nazir, M. A. Khan, S. Anwar, H. Khan, and M. Nazir, "A novel fuzzy logic based software component selection modeling," in Proceedings of the 2012 International Conference on Information Science and Applications (ICISA), pp. 1-6, Suwon, South Korea, May 2012.

[55] S. Nazir, S. Shahzad, S. Mahfooz, and M. Nazir, "Fuzzy logic based decision support system for component security evaluation," The International Arab Journal of Information Technology, vol. 15, no. 2, pp. 224-231, 2018.

[56] S. Tilley and B. Floss, "Hard problems in software testing solutions using testing as a service (TaaS)," vol. 2, no. 1, pp. 1-103, 2014.

[57] W. Kim, S. D. Kim, E. Lee, and S. Lee, “Adoption issues for cloud computing," in Proceedings of the 11th International Conference on Information Integration and Web-Based Applications and Services, pp. 3-6, Kuala Lumpur, Malaysia, December 2009. 
[58] P. Prodanovic, Fuzzy Set Ranking Methods and Multiple Expert Decision Making, Department of Civil and Environmental Engineering Facility for Intelligent Decision Support, University of Western, Ontario, Canada, 2001.

[59] T. H. Chang and T. C. Wang, "Using the fuzzy multi-criteria decision making approach for measuring the possibility of successful knowledge management," Information Sciences, vol. 179, no. 13, pp. 355-370, 2009.

[60] A. Sangaiah and A. Thangavelu, "An exploration of FMCDM approach for evaluating the outcome/success of GSD projects," Central European Journal of Engineering, vol. 3, pp. 419-435, 2013.

[61] G. Li, G. Kou, C. Lin, L. Xu, and Y. Liao, "Multi-attribute decision making with generalized fuzzy numbers," Journal of the Operational Research Society, vol. 66, pp. 1793-1803, 2015.

[62] S. Guo and H. Zhao, "Fuzzy best-worst multi-criteria decision-making method and its applications," Knowledge-Based Systems, vol. 121, pp. 23-31, 2017.

[63] G. Li, G. Kou, and Y. Peng, "A group decision making model for integrating heterogeneous information," IEEE Transactions on Systems, Man, and Cybernetics, vol. 48, no. 6, pp. 1-11, 2016.

[64] G. Kou, Y. Peng, and G. Wang, "Evaluation of clustering algorithms for financial risk analysis using MCDM methods," Information Sciences, vol. 275, pp. 1-12, 2014.

[65] M.-S. Kuo and G.-S. Liang, "A soft computing method of performance evaluation with MCDM based on interval-valued fuzzy numbers," Applied Soft Computing, vol. 12, no. 1, pp. 476-485, 2012.

[66] Y.-J. Wang, "Applying FMCDM to evaluate financial performance of domestic airlines in Taiwan," Expert Systems with Applications, vol. 34, no. 3, pp. 1837-1845, 2008.

[67] L. A. Zadeh, "Fuzzy sets," Information and Control, vol. 8, no. 3, pp. 338-353, 1965.

[68] C. C. Kitchenham, "Guidelines for performing systematic literature reviews in software engineering," Ver. 2.3 EBSE Technical Report, Durham University, Durham, UK, 2007.

[69] T. C. Lethbridge, S. E. Sim, and J. Singer, "Studying software engineers: data collection techniques for software field studies," Empirical Software Engineering, vol. 10, no. 3, pp. 311-341, 2005.

[70] J. W. Creswell, Research Design: Qualitative, Quantitative, and Mixed Methods Approaches, Sage Publications, Thousand Oaks, CA, USA, 2013.

[71] P. Priyadarshinee, R. D. Raut, M. K. Jha, and S. S. Kamble, "A cloud computing adoption in Indian SMEs: scale development and validation approach," The Journal of High Technology Management Research, vol. 28, no. 2, pp. 221-245, 2017.

[72] R. K. Yin, Case Study Research: Design and Methods, Sage Publications, Thousand Oaks, CA, USA, 2009.

[73] M. K. Daskalantonakis, "Achieving higher SEI levels," IEEE Software, vol. 11, no. 4, pp. 17-24, 1994.

[74] S. Tilley and T. Parveen, "SMART-T: migrating testing to the cloud," Software Testing in the Cloud: Migration and Execution, Springer Briefs in Computer Science, pp. 19-35, Springer, Berlin, Germany, 2012.

[75] A. O. Portillo-Dominguez, M. Wang, J. Murphy, and D. Magoni, "Automated WAIT for cloud-based application testing," in Proceedings of the IEEE Seventh International Conference on Software Testing, Verification and Validation Workshops (ICSTW), pp. 370-375, Cleveland, OH, USA, March 2014.

[76] A. Gambi, W. Hummer, and S. Dustdar, "Automated testing of cloud-based elastic systems with AUToCLES," in
Proceedings of the 28th IEEE/ACM International Conference on Automated Software Engineering (ASE), pp. 714-717, Palo Alto, CA, USA, November 2013.

[77] M. Arslan, U. Qamar, S. Hassan, and S. Ayub, "Automatic performance analysis of cloud based load testing of webapplication \& its comparison with traditional load testing," in Proceedings of the 37th International Conference on Software Engineering (ICSE), pp. 140-144, Firenze, Italy, May 2015.

[78] Z. Yang, M. Xiao, X. Zhao, D. Feng, L. Zhang, and H. Song, "Cloud computing and exploration of its application to test field and PHM," in Proceedings of the Prognostics and System Health Management Conference, pp. 1-4, Chengdu, China, October 2016.

[79] H. Muccini, A. D. Francesco, and P. Esposito, "Software testing of mobile applications: challenges and future research directions," in Proceedings of the 7th International Workshop on Automation of Software Test (AST), pp. 29-35, Zurich, Switzerland, June 2012.

[80] Capgemini and Sogetti, "World quality report 2014-2015," 2020, https://www.sogeti.com/explore/reports/world-qualityreport-2019.

[81] J. Zhou, S. Li, Z. Zhang, and Z. Ye, "Position paper: cloudbased performance testing: issues and challenges," in Proceedings of the 2013 International Workshop on Hot Topics in Cloud Services, pp. 55-62, Prague, Czech Republic, April 2013.

[82] L. Ciortea, C. Zamfir, S. Bucur, V. Chipounov, and G. Candea, "Cloud 9," ACM SIGOPS Operating Systems Review, vol. 43, no. 4, pp. 5-10, 2010.

[83] Q. Gao, W. Wang, G. Wu, X. Li, J. Wei, and H. Zhong, "Migrating load testing to the cloud: a case study," in Proceedings of the IEEE Seventh International Symposium on Service-Oriented System Engineering (SOSE), pp. 429-434, Redwood City, CA, USA, March 2013.

[84] S. U. Khan, M. Niazi, and R. Ahmad, "Systematic literature review protocol for a software outsourcing vendors readiness model (SOVRM)," in Proceedings of the International Conference on Information and Communication Technology (ICICT), pp. 1-8, Bannu, Pakistan, 2008.

[85] A. S. Al-Ahmad, S. A. Aljunid, and A. S. A. Sani, "Mobile cloud computing testing review," in Proceedings of the 2013 International Conference on Advanced Computer Science Applications and Technologies (ACSAT), pp. 176-180, Kuching, Malaysia, 2013.

[86] R. D. Raut, P. Priyadarshinee, B. B. Gardas, and M. K. Jha, "Analyzing the factors influencing cloud computing adoption using three stage hybrid SEM-ANN-ISM (SEANIS) approach," Technological Forecasting and Social Change, vol. 134, pp. 98-123, 2018.

[87] S. Ali, L. Hongqi, S. U. Khan, Y. Zhongguo, and Z. Liping, "Success factors for software outsourcing partnership management: an exploratory study using systematic literature review," IEEE Access, vol. 5, pp. 23589-23612, 2017.

[88] S. Ali and S. U. khan, "Critical success factors for software outsourcing partnership (SOP): a systematic literature review," in Proceedings of the 14th International Conference on Global Software Engineering (ICGSE), pp. 153-162, Shangai, China, August 2014.

[89] H. Coolican, Research Methods and Statistics in Psychology, Routledge, London, UK, 7th edition, 2018. 